\title{
Regularity results for the ordinary product stochastic pressure equation
}

\author{
Juan Galvis* $\quad$ Marcus Sarkis ${ }^{\dagger}$
}

October 15, 2011

\begin{abstract}
We consider a stochastic pressure equation with lognormal coefficient with infinite dimensional noise. Using a White Noise framework, we study spatial and stochastic regularity of solutions of the stochastic pressure equation. We first establish that a particular class of weighted chaos spaces can be characterized by Gaussian Sobolev type norms in the random argument under the Gaussian measure. Then, we use these results to prove that the solution of the stochastic pressure equation has the classical regularity in the spatial variable and a stochastic regularity on this class of weighted chaos spaces.
\end{abstract}

Keywords: Stochastic pressure equation, White Noise Analysis, Stochastic Analysis, Weighted chaos norms, Gaussian Sobolev norms. 60H15, $65 \mathrm{~N} 30,60 \mathrm{H} 40,60 \mathrm{H} 07,60 \mathrm{H} 30$

\footnotetext{
${ }^{0}$ AMS subject classifications: 60H30, 60H35, 65C20, 65C30, 65N30

*Department of Mathematics, Texas A \& M University, College Station, TX 77843. email:jugal@math.tamu.edu

${ }^{\dagger}$ Department of Mathematical Sciences, Worcester Polytechnic Institute, Worcester, MA 01609-2280, USA, and Instituto Nacional de Matemática Pura e Aplicada (IMPA), Estrada Dona Castorina 110, Rio de Janeiro-RJ, 22460-320, Brazil. e-mail:msarkis@wpi.edu
} 


\section{Contents}

1 Introduction $\quad 2$

2 Framework: White noise analysis $\quad 6$

3 Smooth random functions $\quad 7$

3.1 Wiener-Chaos expansion ............... 7

3.2 Weighted chaos norms . . . . . . . . . . . . . 8

3.3 Derivatives and weighted chaos norms . . . . . . . . . . 9

3.3.1 Chaos weighted norms and the operator $\Gamma_{\oplus}(A)$. . . . 9

3.3.2 Derivatives and Gaussian Sobolev norms . . . . . . . . . . 9

3.4 Equivalence of norms . . . . . . . . . . . . . . . . 11

3.4.1 A remark on Kondratiev type norms . . . . . . . . . 16

4 Application to elliptic equations with lognormal coefficients 17

4.1 Examples and comments . . . . . . . . . . . . . . 17

4.2 Dealing with non-uniform ellipticity using a white noise approach 18

4.3 Galerkin approximation and a priori error estimates using Weighted norms ...................... 20

4.4 Stochastic regularity . . . . . . . . . . . . . . . . . . . 22

4.5 Spatial regularity . . . . . . . . . . . . . . . 29

5 Final remarks

\section{Introduction}

Uncertainty quantification techniques have gained the attention of researches in the last years. The theoretical and numerical treatments of stochastic partial differential equations are important for uncertainty quantification because the behavior of many interesting random quantities is described by partial differential equations. In particular, we study elliptic partial differential equations which are important for the better understanding of many physical and engineering systems. In this paper we study regularity results for the equation

$$
\left\{\begin{aligned}
-\nabla_{x} \cdot\left(\kappa(x, \omega) \nabla_{x} u(x, \omega)\right) & =f(x, \omega), \text { for all } x \in \mathcal{O} \\
u(x, \omega) & =0, \text { for all } x \in \partial \mathcal{O}
\end{aligned}\right.
$$

where $\log \kappa(x, \omega)$ is a Gaussian field and $f$ is a (possible random) forcing term. Some important questions related to (1) are: existence and uniqueness of solutions, design of efficient numerical methods, a priori and a posteriori error estimates for suitable numerical strategies and regularity results in the $\omega$ variable, among others. Regularity results in the spatial variable and the stochastic variable are important in order to design and validate numerical methods for this problem. In this paper we study spatial and stochastic regularity of solutions of (1). The analysis presented here complement our previous paper [14] where we 
address existence and uniqueness of solutions of (1) and general a priori error estimates for finite element approximations. It is important to note that the presence of the log-normal coefficient $\kappa(x, \cdot)$ induces lack of uniform ellipticity and boundedness, and therefore, the analysis become very challenging.

When studying existence of solutions, error estimates for, or regularity of (1), another level of difficulty is the infinite dimensional behavior of the Gaussian fields. The need for infinite dimensional analysis is motivated by applications as explained below. We also note that the assumption of finite dimensional noise, compared to the infinite dimensional noise case, in the coefficient $\kappa$ and the forcing term $f$ simplify the analysis a great deal, however, it has serious practical limitations. Indeed, the dimension of the finite dimensional noise is often associated with truncated or finite dimensional approximations of Karhunen-Loève (KL) expansions or chaos expansions. In some real-world applications the dimension of the noise may be very large, for instance, in applications related to flow in heterogeneous porous media. In this case, the coefficient $\kappa$ represents the permeability of a porous medium that contain uncertainties at the fine resolution. Since permeability data are also collected at finest scales, such as core scales, detailed geological models are constructed to contain such scales. At these scales, we have to deal with large uncertainties associated with the fine grid information. Modeling this detailed geological system may require large dimensional set to parametrize the noise. Due to the high-computational cost, it may not be possible to compute using the high noise dimension and lower dimensional models need to be used. Hence, robust error estimates and analysis, for lower dimensional stochastic discretizations, that take into account these fine-scale uncertainties (not included in the discretized model), are needed. In this case, it is more advantageous to work with infinite dimensional stochastic space due to a large dimension of the stochastic space. The White Noise analysis is a suitable framework to develop this infinite dimensional analysis. In this paper, the case of infinite dimensional noise is considered.

We mention some related works and models. We refer to $[1,7,30]$ and references there in for the study of general elliptic equations with bounded coefficients and finite dimensional noise assumption. In particular, in [7], the author obtains regularity of solutions in the random argument (only) in general Sobolev spaces. The results in [7] hold for general bounded coefficients with bounded derivatives in the random arguments.

Regarding the existence of solutions and its numerical approximation, there are few works addressing the difficulty of the lack of uniform ellipticity and boundedness. We mention the works [14, 27] where the White Noise framework analysis is carried out in the framework of Hilbert spaces which allows the use of classical finite element methods. In particular, in [14] (2009), among other results, it is established the existence and uniqueness of solution in Hilbert spaces with square norm given by $\int\|u(x, \omega)\|_{H_{0}^{1}}^{2} \gamma(\omega) d \mu(\omega)$ where $\mu$ is the standard Gaussian measure and $\gamma(\omega)$ represent a weight properly chosen (that includes the case $\gamma=1)$. The resulting measure $\gamma(\omega) d \mu(\omega)$ can be view as and infinite product of one dimensional Gaussian measures with some given variances. 
See [14]. Studies of regularity of solutions are not considered in [14]. In the work [15] (2010), among other results, the author obtain existence of solutions. The author obtain existence of solutions using Hilbert norms with square given by $\int\|u(x, \omega)\|^{2} d \hat{\gamma}(\omega)$ where $\hat{\gamma}$ is a infinite product of one dimensional Gaussian measures. The measure $\hat{\gamma}$ is different from the standard Gaussian measure $\mu$. The author also obtain existence of solutions using Banach norms of the form $\left(\int\|u(x, \omega)\|^{p} d \mu(\omega)\right)^{1 / p}$ with $p \neq 2$. This work does not study regularity of the solutions. In a recent work, [9], the author obtain existence of solutions using norms of the form $\left(\int\|u(x, \omega)\|^{p} d \widetilde{\gamma}(\omega)\right)^{1 / p}$ where $p>0$ and $\widetilde{\gamma}$ is the measure XXXpreciso ajuda aqui, acho que vou escrever para a Julia e perguntar que medida eh esta? 0 ve acha melhor nao dizer nada.

Some researchers have consider Wick product, instead of ordinary product, in (1). In $[2,29]$ the authors consider a Wick product formulation, and in $[23,33,32]$ weighted Wiener chaos expansions and other modeling methodology are proposed. Regularity results for Wick product pressure equations with log-normal coefficient have been considered for several authors. We emphasize that in (1) we use the ordinary product $\kappa(x, \omega) \nabla_{x} u(x, \omega)$ rather then the Wick product, $\kappa(x, \omega) \diamond \nabla_{x} u(x, \omega)$. For regularity results of stochastic pressure equations of Wick type see [3] and references therein. We also mention [32] were new ways of introducing the Wick calculus in the pressure equation are explored. In [3], the authors find the chaos expansion of the solution of the Wick product pressure equation, and calculate its stochastic regularity in the distributional sense using Kondratiev type norms, see [20, 18]. One of the main properties of the Wick product is that it simplifies the computation of chaos expansion of the Wick product of two random functions when compared with the ordinary product. Indeed, the $n$-th order coefficient of the Wick product of two random functions is the product of the corresponding $n$-th order coefficient of the two random functions involved. This simplification allows the computation of the Chaos expansion of the solution of the Wick product pressure equation.

In this paper we study joint spatial and stochastic regularity for (1). The results we present here complement and build up on our previous paper [14] that we describe next. A more detailed review is presented in Section 4. In [14], it is considered the Problem (1) where $\kappa(x, \omega):=e^{W_{\phi}(x, \omega)}=e^{\left\langle\omega, \phi_{x}\right\rangle}, x \in \mathcal{O}, \omega \in \mathcal{S}^{\prime}$ and the exponent $W_{\phi}(x, \omega)$ is the smoothed White Noise process defined on the White Noise probability space $\left(\mathcal{S}^{\prime}, \mathcal{B}\left(\mathcal{S}^{\prime}\right), \mu\right)$ and well-posedness results were established in tensor product of Hilbert spaces. Here $\mathcal{S}^{\prime}$ is an appropriately chosen space of distributions; see Section 2 below. The tensor product space of the solution involves the usual $H_{0}^{1}(\mathcal{O})$ space and the Hilbert space $\left(L^{2}\right)_{s}$ (a weighted $L^{2}$ space in $\mathcal{S}^{\prime}$ with a exponential type weight). The parameter $s$ is related to the stochastic exponential decay (or growth) of the functions on the White Noise probability space. The $\left(L^{2}\right)_{s}$ space has two fundamental aspects, it circumvents the lack of uniform ellipticity and boundedness of the problem, and due to its Hilbert space structure, its norm can be computed easily using orthogonality relations; see Section 3.1 below. Furthermore, in the resulting tensor product space we can use orthogonal projections to analyze finite dimensional Galerkin type methods and to obtain a priori error estimates; 
see Section 4.3 below. For the a priori error estimates, as usual, some regularity of the solution need to be assumed. It is assumed that the solution of (1) to belong to a more regular tensor product space (in $x$ and $\omega$ ). The regular tensor space used in [14] involves the spaces $H^{2}(\mathcal{O})$, for the regularity in the $x$ variable, and a weighted chaos space for the regularity in the $\omega$ variable. For different weighted chaos norms we cite $[3,6,10,14,16,17,18,20,22,26]$ and references therein. The weighted chaos spaces norms depend on the choice of a sequence of weights. The corresponding norm measures the decay of the coefficients in the chaos expansion of a random function. We recall that the chaos expansion of a random function is its expansion in terms of Fourier-Hermite orthogonal polynomials. The a priori error estimates in [14] are general and apply to any weighted chaos space. In [14], no studies concerning regularity beyond $\left(L^{2}\right)_{s} \otimes H^{1}(\mathcal{O})$ are obtained.

In this paper we study the joint spatial and stochastic regularity of solutions of (1) assuming similar regularity for the right-hand side $f(x, \omega)$ and the smoothed White Noise $W_{\phi}(x, \omega)$. A main issue is that the computation of the weighted chaos norms turn out to be difficult when the chaos expansion of the solution is not available. For solutions of (1), it is difficult to write a manageable expression for the chaos expansion of the solution, either in terms of FourierHermite polynomials or in terms of multiple Itô integrals, see $[16,18,26]$. On the other hand, Gaussian Sobolev spaces have been also used in the literature, $[11,24,28]$. The Gaussian Sobolev norms involve $\left(L^{2}\right)_{s}$ norms of (Gâteaux) derivatives of random functions. We prove that a particular weighted chaos (use in [14] to obtain a priori error estimates) can be characterized using Sobolev type norms in the $\omega$ variable for the Gaussian measure as in [11, 28, 24]; see Theorem 12. This equivalence of norms is useful to obtain regularity results for this norms. It is easier to obtain bounds for partial derivatives (in the stochastic variable $\omega$ ) than obtaining bounds for the coefficients of the chaos expansions of the solution of (1). Our main result is given in Theorem 30 where we prove that the solution of (1) has regularity $H^{2}$ in the spatial variable $x$, see Lemma 27, and stochastic regularity given by a particular weighted chaos space, see Lemma 20. In particular, the weighted chaos spaces used in Theorem 30 require norms of partial (or Gâteaux) derivatives in the $\omega$ variable up to certain order to be bounded.

In Section 2 we introduce the White Noise framework to be used in the paper. In Section 3 we present the study of smooth random functions. We define the weighted chaos norms to be used in the paper. Sections 3.3 and 3.4 are dedicated to the use of (Gâteaux) derivatives in the $\omega$ variable to compute weighted chaos norms. Section 4 is dedicated to apply the results from Section 3 to infinity-dimensional noise elliptic problem with log-normal coefficients. In Sections 4.1, 4.2 and 4.3 review useful results from [14] to be used later and introduce some notations for tensor product functions spaces. In Section 4.4 we study the stochastic regularity of solutions and in Section 4.5 we obtain also the spatial regularity results. Final remarks are presented in Section 5. 


\section{Framework: White noise analysis}

Let $H$ be a real Hilbert space with inner product $(\cdot, \cdot)_{H}$ and norm $\|\cdot\|_{H}$, and let $A$ be an operator on $H$ such that there exists an $H$-orthonormal basis $\left\{\eta_{j}\right\}_{j=1}^{\infty}$ satisfying (see Examples 15 and 16 below):

1) $A \eta_{j}=\lambda_{j} \eta_{j}, j=1,2, \ldots$

2) $1<\lambda_{1} \leq \lambda_{2} \leq \cdots$.

3) $\sum_{j=1}^{\infty} \lambda_{j}^{-2 \theta}<\infty$ for some constant $\theta>0$.

For $p>0$ let $\mathcal{S}_{p}:=\left\{\xi \in H ;\|\xi\|_{p}<\infty\right\}$ where

$$
\|\xi\|_{p}^{2}:=\left\|A^{p} \xi\right\|_{H}^{2}=\sum_{j=0}^{\infty} \lambda_{j}^{2 p}\left(\xi, \eta_{j}\right)_{H}^{2},
$$

and for $p<0$ let $\mathcal{S}_{p}$ be defined as the dual space of $\mathcal{S}_{-p}$. It is easy to see that for $p<0$ we also have $\|\cdot\|_{p}=\left\|A^{p} \cdot\right\|_{H}$ and the duality pairing between $\mathcal{S}_{p}$ and $\mathcal{S}_{-p}$ is an extension of the $H$ inner product. We also define

$$
\mathcal{S}=\cap_{p \geq 0} \mathcal{S}_{p} \text { (with the projective limit topology) }
$$

and let $\mathcal{S}^{\prime}$ be defined as the dual space of $\mathcal{S}$, i.e., by considering the standard countably Hilbert space constructed from $(H, A)$; see $[20,26]$.

Let $\mathcal{S}^{\prime}$ be the probability space with the sigma-field $\mathcal{B}\left(\mathcal{S}^{\prime}\right)$ of Borel subsets of $\mathcal{S}^{\prime}$. The probability measure $\mu$ is given by the Bochner-Minlos theorem and characterized by

$$
E_{\mu} e^{i\langle\cdot \xi\rangle}:=\int_{\mathcal{S}^{\prime}} e^{i\langle\omega, \xi\rangle} d \mu(\omega)=e^{-\frac{1}{2}\|\xi\|_{H}^{2}}, \text { for all } \xi \in \mathcal{S} .
$$

Here, the pairing $\langle\omega, \xi\rangle=\omega(\xi)$ is the action of $\omega \in \mathcal{S}^{\prime}$ on $\xi \in \mathcal{S}$, and $E_{\mu}$ denotes the expectation with respect to the measure $\mu$. See $[5,16,17,18,20,26]$ and references therein for details on the Bochner-Minlos theorem, the measure $\mu$ and (2). The measure $\mu$ is often called the (normalized) Gaussian measure on $\mathcal{S}^{\prime}$. We note that from (2) we have that for any function $\xi \in H$, the random variable $\omega \mapsto\langle\omega, \xi\rangle$ can be defined in the $L^{2}(\mu)$ sense and it is normally distributed with zero mean and variance $\|\xi\|_{H}^{2}$; see $[18,20,26]$.

The following particular case of Fernique's Theorem will be used throughout this paper; see Lemma 2.2 in [14] for he proof and $[11,12,19,28]$ for the general case.

Lemma 1. We have

$$
\int_{\mathcal{S}^{\prime}} e^{s\|\omega\|_{-\theta}^{2}} d \mu(\omega)= \begin{cases}\prod_{j=1}^{\infty}\left(1-\frac{2 s}{\lambda_{j}^{2 \theta}}\right)^{-\frac{1}{2}}, & s<\frac{\lambda_{1}^{2 \theta}}{2} \\ +\infty, & s \geq \frac{\lambda_{1}^{2 \theta}}{2} .\end{cases}
$$


We note that Lemma 1 implies that $\int_{\mathcal{S}^{\prime}}\|\omega\|_{-\theta}^{2} d \mu<\infty$ which in turn implies that $\mu\left(\mathcal{S}_{-\theta}\right)=1$. To see this, note that $\mathcal{S}^{\prime} \backslash \mathcal{S}_{-\theta}=\left\{\omega:\|\omega\|_{-\theta}^{2}=\infty\right\}$ and then $\mu\left(\mathcal{S}^{\prime} \backslash \mathcal{S}_{-\theta}\right)>0$ would imply that $\int_{S^{\prime}}\|\omega\|_{-\theta}^{2} d \mu=\infty$ which gives a contradiction. Without further comments, we use that $\mu\left(\mathcal{S}_{-\theta}\right)=1$ throughout this paper.

In what follows we use the notation $\left(L^{2}\right)$ for the space $L^{2}(\mu)$. We always interpret properties in the "almost surely" sense (with respect to $\mu$ ), therefore, we will sometimes omit this interpretation to make notation and formula less cumbersome.

\section{Smooth random functions}

We introduce the space

$$
\left(L^{2}\right)_{s}:=L^{2}\left(\mathcal{S}^{\prime}, e^{s\|\omega\|_{-\theta}^{2}} d \mu(\omega)\right)
$$

with norm $\|v\|_{\left(L^{2}\right)_{s}}^{2}:=\int_{\mathcal{S}^{\prime}}|v(\omega)|^{2} e^{s\|\omega\|_{-\theta}^{2}} d \mu$. In [14] we established the existence of solutions of $(1)$ in the tensor product space $H_{0}^{1}(\mathcal{O}) \otimes\left(L^{2}\right)_{s}$. See Section 4 for a review of these results. In this section we introduce subspaces of $\left(L^{2}\right)_{s}$ consisting of smooth functions. These subspaces of smooth functions will be used in Section 4 to obtain regularity results for problem (1).

\subsection{Wiener-Chaos expansion}

In the following we characterize the space $\left(L^{2}\right)_{s}$ defined in $(3)$. We need to consider multi-index of arbitrary length. To simplify the notation, we regard multi-indices as elements of the space $\left(\mathbb{N}_{0}^{\mathbb{N}}\right)_{c}$ of all sequences $\boldsymbol{\alpha}=\left(\alpha_{1}, \alpha_{2}, \ldots\right)$ with elements $\alpha_{j} \in \mathbb{N}_{0}=\mathbb{N} \cup\{0\}$ and with compact support, i.e., with only finitely many $\alpha_{j} \neq 0$. We write $\mathcal{J}=\left(\mathbb{N}_{0}^{\mathbb{N}}\right)_{c}$. Given $\boldsymbol{\alpha} \in \mathcal{J}$ define the order and length of $\boldsymbol{\alpha}$, denoted by $d(\boldsymbol{\alpha})$ and $|\boldsymbol{\alpha}|$ respectively, by

$$
d(\boldsymbol{\alpha})=\max \left\{j: \alpha_{j} \neq 0\right\} \quad \text { and }|\boldsymbol{\alpha}|=\alpha_{1}+\alpha_{2}+\ldots+\alpha_{d(\boldsymbol{\alpha})} .
$$

We also introduce the $\sigma$-Hermite polynomials, $h_{\sigma^{2}, n}$, where $\sigma>0$ and $n=$ $0,1,2, \ldots$ These polynomials can be defined by the generating function identity

$$
e^{t x-\frac{1}{2} \sigma^{2} t^{2}}=\sum_{n=0}^{\infty} \frac{t^{n}}{n !} h_{\sigma^{2}, n}(x) .
$$

When $\sigma^{2}=1$ we denote $h_{1, n}$ simply by $h_{n}$. Note that $h_{\sigma^{2}, n}(x)=\sigma^{n} h_{n}(x / \sigma)$ and $h_{\sigma^{2}, n}^{\prime}(x)=n h_{\sigma^{2}, n-1}(x)$. The $\sigma$-Hermite polynomials are an orthogonal basis for $L^{2}\left(\mathbb{R}, e^{-\frac{1}{2 \sigma^{2}} x^{2}} d x\right)$.

For $s<\frac{\lambda_{1}^{2 \theta}}{2}$ define $\sigma_{j}=\sigma_{j}(s)=\left(1-\frac{2 s}{\lambda_{j}^{2 \theta}}\right)^{-\frac{1}{2}}, j=1,2, \ldots$, and for $\boldsymbol{\alpha} \in \mathcal{J}$ let $\boldsymbol{\sigma}^{\boldsymbol{\alpha}}=\boldsymbol{\sigma}^{\boldsymbol{\alpha}}(s):=\prod_{j=1}^{d(\boldsymbol{\alpha})} \sigma_{j}^{\alpha_{j}}(s)$ and $\sigma_{*}=\sigma_{*}(s):=\int_{\mathcal{S}^{\prime}} e^{s\|\omega\|_{-\theta}^{2}} d \mu(\omega)$. From Lemma $1, \sigma_{*}=\prod_{j=1}^{\infty} \sigma_{j}<\infty$ when $s<\frac{\lambda_{1}^{2 \theta}}{2}$. Now we define the $\boldsymbol{\sigma}(s)$-FourierHermite polynomials. 
Definition 2. Given $s<\frac{\lambda_{1}^{2 \theta}}{2}, \boldsymbol{\alpha}=\left(\alpha_{1}, \alpha_{2}, \ldots\right) \in \mathcal{J}$ and $\boldsymbol{\sigma}=\boldsymbol{\sigma}(s)=$ $\left(\sigma_{1}, \sigma_{2}, \ldots\right)$, define

$$
H_{\boldsymbol{\sigma}^{2}, \boldsymbol{\alpha}}(\omega)=\frac{1}{\sqrt{\sigma_{*}}} \prod_{j=1}^{d(\boldsymbol{\alpha})} h_{\sigma_{j}^{2}, \alpha_{j}}\left(\left\langle\omega, \eta_{j}\right\rangle\right) ; \quad \omega \in \mathcal{S}^{\prime} .
$$

We now state the Wiener-Chaos expansion theorem; see $[11,16,18,17,26]$ for the case $s=0$.

Theorem 3. When $s<\frac{\lambda_{1}^{2 \theta}}{2}$, the $\boldsymbol{\sigma}(s)$-Fourier-Hermite polynomials are orthogonal in $\left(L^{2}\right)_{s}$. Moreover,

$$
\left\|H_{\boldsymbol{\sigma}^{2}(s), \boldsymbol{\alpha}}\right\|_{\left(L^{2}\right)_{s}}^{2}=\boldsymbol{\alpha} ! \boldsymbol{\sigma}(s)^{2 \boldsymbol{\alpha}} .
$$

In addition, every polynomial in $\omega$ belongs to $(L)_{s}$ and every $u \in\left(L^{2}\right)_{s}$ can be represented as a Wiener-Chaos expansion

$$
u=\sum_{\boldsymbol{\alpha} \in \mathcal{J}} u_{\boldsymbol{\alpha}, s} H_{\boldsymbol{\sigma}(s)^{2}, \boldsymbol{\alpha}} \quad \text { with }\|u\|_{\left(L^{2}\right)_{s}}^{2}=\sum_{\boldsymbol{\alpha} \in \mathcal{J}} \boldsymbol{\alpha} ! \boldsymbol{\sigma}(s)^{2 \boldsymbol{\alpha}} u_{\boldsymbol{\alpha}, s}^{2} .
$$

\subsection{Weighted chaos norms}

Now we introduce weighted chaos norms that are used to define subspaces of $\left(L^{2}\right)_{s}$ consisting of smooth functions.

In $\left(L^{2}\right)_{s}$ with $s<\frac{\lambda_{1}^{2 \theta}}{2}$ we introduce the system of Hilbert norms

$$
\|u\|_{p ; \rho, s}^{2}:=\sum_{\boldsymbol{\alpha} \in \mathcal{J}} \rho(\boldsymbol{\alpha}, p)^{2} \boldsymbol{\alpha} ! \boldsymbol{\sigma}(s)^{2 \boldsymbol{\alpha}} u_{\boldsymbol{\alpha}, s}^{2},
$$

where $u=\sum_{\boldsymbol{\alpha} \in \mathcal{J}} u_{\boldsymbol{\alpha}, s} H_{\sigma(s)^{2}, \boldsymbol{\alpha}}$. We assume that $\rho(\boldsymbol{\alpha}, q) \geq \rho(\boldsymbol{\alpha}, p)>0$ for all $q>p \geq 0$ and that $\rho(\boldsymbol{\alpha}, 0)=1$ for all $\boldsymbol{\alpha} \in \mathcal{J}$. Usually, the weights $\rho(\boldsymbol{\alpha}, p)$ are the eigenvalues of some nonnegative operator in $\left(L^{2}\right)_{s}$ with the $\sigma(s)$-FourierHermite polynomials as eigenfunctions; see [18, 17, 26, 20, 6, 10, 3] for the case $s=0$.

For $p>0$ define the spaces $\mathcal{S}_{p, \rho, s}$ by

$$
\mathcal{S}_{p ; \rho, s}=\left\{v \in\left(L^{2}\right)_{s}:\|v\|_{p ; \rho, s}<\infty\right\} .
$$

For $p<0$ define $\mathcal{S}_{p, \rho, s}$ as the dual space of $\mathcal{S}_{-p ; \rho, s}$. We have $\mathcal{S}_{0 ; \rho, s}=\left(L^{2}\right)_{s}$ and the inclusion $\mathcal{S}_{q ; \rho, s} \subset \mathcal{S}_{p ; \rho, s}$ holds for all $q>p$.

Given a multi-index $\boldsymbol{\alpha}$ we denote $\langle\boldsymbol{\alpha}, \boldsymbol{\lambda}\rangle:=\sum_{j=1}^{d(\boldsymbol{\alpha})} \alpha_{j} \lambda_{j}$. Note that we have $\langle\boldsymbol{\alpha}, \boldsymbol{\lambda}\rangle \geq 0$. We consider the following weight (see $[6,11,17,24,28]$ )

$$
\rho(\boldsymbol{\alpha}, p)^{2}=1+\langle\boldsymbol{\alpha}, \boldsymbol{\lambda}\rangle^{2 p}, \quad p>0, \text { and } \rho(\boldsymbol{\alpha}, 0)=1, \quad \boldsymbol{\alpha} \in \mathcal{J} .
$$

Alternatively, the equivalent weight $\rho(\boldsymbol{\alpha}, p)=(1+\langle\boldsymbol{\alpha}, \boldsymbol{\lambda}\rangle)^{p}$ can be used. Some of the algebra is simpler using the weight in (7). See Section 3.3.1. For examples of other weights $\rho(\boldsymbol{\alpha}, p)$ we refer to $[11,6,16,18,20,26,24,28]$. See also Section 3.4.1 for a remark on Kondratiev type weights. 


\subsection{Derivatives and weighted chaos norms}

We showed in [14] that weighted chaos norms are appropriate in order to obtain a priori error estimates for Finite Element approximations of (1). The error estimates in [14] assume that weighted chaos norms of the solution of (1) are bounded. This is one main motivation for regularity studies carried out in this paper; see Section 4 below. It turns out that the weighted norms in (5) are, in general, difficult to compute or estimate when the chaos expansion is not explicitly available. In this section we prove that some chaos weighted norms can be computed using $\left(L^{2}\right)_{s}$ norms of partial derivatives; see Section 3.4. This is a main step for our regularity studies since obtaining norms of derivative (with respect to $\omega$ ) of the solution of (1) is an easier task than obtaining bounds for weighted chaos norms; see Lemma 20 in Section 4.

Next, in Section 3.3.1 we recall that the weighted norms can be written as a square integral, in the White Noise measure, using an operator acting on functions in $\left(L^{2}\right)_{s}$. In Section 3.3.2 we review the computation of $\left(L^{2}\right)_{s}$ norms of derivatives.

\subsubsection{Chaos weighted norms and the operator $\Gamma_{\oplus}(A)$}

We consider the weighted norm (5) with the particular weight in (7). We can write

$$
\begin{aligned}
\|u\|_{p ; \rho, s}^{2} & =\sum_{\boldsymbol{\alpha} \in \mathcal{J}}\left(1+\langle\boldsymbol{\alpha}, \boldsymbol{\lambda}\rangle^{2 p}\right) \boldsymbol{\alpha} ! \boldsymbol{\sigma}(s)^{2 \boldsymbol{\alpha}} u_{\boldsymbol{\alpha}, s}^{2}, \\
& =\|u\|_{\left(L^{2}\right)_{s}}^{2}+\left\|\Gamma_{\oplus}(A)^{p} u\right\|_{\left(L^{2}\right)_{s}}^{2} \\
& =\int_{\mathcal{S}^{\prime}}\left(|u(\omega)|^{2}+\left|\Gamma_{\oplus}(A)^{p} u(\omega)\right|^{2}\right) e^{s\|\omega\|_{-\theta}^{2}} d \mu(\omega),
\end{aligned}
$$

where $\Gamma_{\oplus}(A)$ is the operator defined by

$$
\Gamma_{\oplus}(A) H_{\boldsymbol{\sigma}^{2}, \boldsymbol{\alpha}}=\langle\boldsymbol{\alpha}, \boldsymbol{\lambda}\rangle H_{\boldsymbol{\sigma}^{2}, \boldsymbol{\alpha}} .
$$

We point out that $\Gamma_{\oplus}\left(A^{p}\right) \neq \Gamma_{\oplus}(A)^{p}$ since $\Gamma_{\oplus}(A)^{p} H_{\boldsymbol{\sigma}^{2}, \boldsymbol{\alpha}}=\langle\boldsymbol{\alpha}, \boldsymbol{\lambda}\rangle^{p} H_{\boldsymbol{\sigma}^{2}, \boldsymbol{\alpha}}$ and $\Gamma_{\oplus}\left(A^{p}\right) H_{\boldsymbol{\sigma}^{2}, \boldsymbol{\alpha}}=\left\langle\boldsymbol{\alpha}, \boldsymbol{\lambda}^{p}\right\rangle H_{\boldsymbol{\sigma}^{2}, \boldsymbol{\alpha}}$. We observe that $\left\|\Gamma_{\oplus}(A)^{p} \cdot\right\|_{\left(L^{2}\right)_{s}}^{2}$ is a norm in the space of function in $\left(L^{2}\right)_{s}$ with $u_{\mathbf{0}}=0$ in its $\sigma(s)$-Fourier-Hermite expansion.

\subsubsection{Derivatives and Gaussian Sobolev norms}

Using partial derivative (as in the deterministic Sobolev spaces norms), we want to be able to compute a norm equivalent to the norm (5) with the weights $\rho$ defined (7).

In this section we work with differential operators acting on $\left(L^{2}\right)_{s}$ and define Sobolev type norms for Gaussian measure; see [6, 11, 17, 20, 24, 28, 31, 34] and references therein. Denote by $\partial_{\ell} u$ the (Gâteaux differential at $\omega$ in the direction 
of $\eta_{\ell}$ or) directional derivative of $u$ in the direction of the $\ell$-th basis function $\eta_{\ell} \in \mathcal{S}$. Given $u \in\left(L^{2}\right)_{s}$ we have

$$
\partial_{\ell} u(\omega):=\left.\frac{d}{d t} u\left(\omega+t \eta_{\ell}\right)\right|_{t=0} .
$$

For any Fourier-Hermite polynomial $H_{\boldsymbol{\sigma}^{2}, \boldsymbol{\alpha}}$ with $\alpha_{\ell}>0$ we have that

$$
\partial_{\ell} H_{\boldsymbol{\sigma}^{2}, \boldsymbol{\alpha}}(\omega)=\partial_{\ell} \prod_{j=1}^{d(\boldsymbol{\alpha})} h_{\sigma_{j}^{2}, \alpha_{j}}\left(\left\langle\omega, \eta_{j}\right\rangle\right)=\alpha_{\ell} H_{\boldsymbol{\sigma}^{2}, \boldsymbol{\alpha}-\boldsymbol{\xi}_{\ell}}(\omega)
$$

where $\boldsymbol{\xi}_{\ell}$ is the multi-index with one in the $\ell$-entry and zero in the other positions so that $\boldsymbol{\alpha}-\boldsymbol{\xi}_{\ell}=\left(\alpha_{1}, \ldots, \alpha_{\ell-1}, \alpha_{\ell}-1, \alpha_{\ell+1}, \ldots\right)$. Here we have used that $h_{\sigma^{2}, n}^{\prime}=n h_{\sigma^{2}, n-1}$, see (4). For $\alpha_{\ell}=0$ define $\partial_{\ell} H_{\boldsymbol{\sigma}^{2}, \boldsymbol{\alpha}}(\omega)=0$. Then for $u=\sum_{\boldsymbol{\alpha} \in \mathcal{J}} u_{\boldsymbol{\alpha}} H_{\boldsymbol{\sigma}^{2}, \boldsymbol{\alpha}}$ such that $\partial_{j} u \in\left(L^{2}\right)_{s}$ we have

$$
\partial_{\ell} u(\omega)=\sum_{\boldsymbol{\alpha} \in \mathcal{J}} \alpha_{\ell} u_{\boldsymbol{\alpha}} H_{\boldsymbol{\sigma}^{2}, \boldsymbol{\alpha}-\boldsymbol{\xi}_{\ell}}(\omega)
$$

and

$$
\left\|\partial_{\ell} u\right\|_{\left(L^{2}\right)_{s}}^{2}=\sum_{\boldsymbol{\alpha} \in \mathcal{J}} \alpha_{\ell}^{2} u_{\boldsymbol{\alpha}}^{2} \boldsymbol{\sigma}^{2 \boldsymbol{\alpha}}\left(\boldsymbol{\alpha}-\boldsymbol{\xi}_{\ell}\right) !=\sum_{\boldsymbol{\alpha} \in \mathcal{J}} \alpha_{\ell} u_{\boldsymbol{\alpha}}^{2} \boldsymbol{\sigma}^{2 \boldsymbol{\alpha}} \boldsymbol{\alpha} !,
$$

where we have used that $\alpha_{\ell}\left(\boldsymbol{\alpha}-\boldsymbol{\xi}_{\ell}\right)$ ! $=\boldsymbol{\alpha}$ !; see [11]. Analogously, for any Fourier-Hermite polynomial the $\boldsymbol{\gamma}$ partial derivative $\partial^{\gamma}$ can be computed as

$$
\begin{aligned}
\partial^{\gamma} H_{\boldsymbol{\sigma}^{2}, \boldsymbol{\alpha}}(\omega) & =\prod_{\ell=1}^{d(\boldsymbol{\gamma})} \partial_{\ell}^{\gamma /} \prod_{j=1}^{d(\boldsymbol{\alpha})} h_{\sigma_{j}^{2}, \alpha_{j}}\left(\left\langle\omega, \eta_{j}\right\rangle\right) \\
& =\prod_{j=1}^{d(\alpha)} \frac{\alpha_{j} !}{\left(\alpha_{j}-\gamma_{j}\right) !} h_{\sigma_{j}^{2}, \alpha_{j}-\gamma_{j}}\left(\left\langle\omega, \eta_{j}\right\rangle\right)=\frac{\boldsymbol{\alpha} !}{(\boldsymbol{\alpha}-\boldsymbol{\gamma}) !} H_{\boldsymbol{\sigma}^{2}, \boldsymbol{\alpha}-\boldsymbol{\gamma}}(13)
\end{aligned}
$$

for every multi-indexes $\boldsymbol{\gamma}$ and $\boldsymbol{\alpha}$ with $\boldsymbol{\gamma} \leq \boldsymbol{\alpha}$. Then for $u=\sum_{\boldsymbol{\alpha} \in \mathcal{J}} c_{\boldsymbol{\alpha}} H_{\boldsymbol{\sigma}^{2}, \boldsymbol{\alpha}}$ we have

$$
\partial^{\gamma} u(\omega)=\sum_{\boldsymbol{\alpha} \geq \boldsymbol{\gamma}} \frac{\boldsymbol{\alpha} !}{(\boldsymbol{\alpha}-\gamma) !} u_{\boldsymbol{\alpha}} H_{\boldsymbol{\sigma}^{2}, \boldsymbol{\alpha}-\boldsymbol{\gamma}}(\omega) .
$$

This implies that the $\left(L^{2}\right)_{s}$ norm of $\partial^{\gamma} u$ is given by

$$
\begin{aligned}
\left\|\partial^{\gamma} u\right\|_{\left(L^{2}\right)_{s}}^{2} & =\sum_{\boldsymbol{\alpha} \geq \boldsymbol{\gamma}} \frac{\boldsymbol{\alpha} !^{2}}{(\boldsymbol{\alpha}-\gamma) !^{2}} u_{\boldsymbol{\alpha}}^{2} \boldsymbol{\sigma}^{2 \boldsymbol{\alpha}}(\boldsymbol{\alpha}-\gamma) ! \\
& =\sum_{\boldsymbol{\alpha} \geq \gamma} \frac{\boldsymbol{\alpha} !}{(\boldsymbol{\alpha}-\gamma) !} u_{\boldsymbol{\alpha}}^{2} \boldsymbol{\sigma}^{2 \boldsymbol{\alpha}} \boldsymbol{\alpha} ! .
\end{aligned}
$$

Remark 4. Recall that when $s=0$ we have $\left\|\partial^{\gamma} u\right\|_{\left(L^{2}\right)}^{2}=\int_{\mathcal{S}^{\prime}}\left|\partial^{\gamma} u(\omega)\right|^{2} d \mu(\omega)$ and we refer to norms defined in terms of $\left(L^{2}\right)$ norms of partial derivatives as Gaussian Sobolev norms. We will use the same terminology for the case $s \neq 0$. 


\subsection{Equivalence of norms}

This section is dedicated to prove that, using partial derivative, we can compute the norm $\|\cdot\|_{\frac{k}{2} ; \rho, s}, k \in \mathbb{N}$, defined in Section 3.2. We will prove in Theorem 12 that for every $k \in \mathbb{N}$ we have

$$
\|u\|_{\frac{k}{2} ; \rho, s}^{2}=\|u\|_{\left(L^{2}\right)_{s}}^{2}+\sum_{i=1}^{k} \sum_{R \in P^{k, i}} \sum_{\ell_{1}, \ell_{2}, \ldots, \ell_{i}} \lambda_{\ell_{1}}^{2 R_{1}} \ldots \lambda_{\ell_{i}}^{2 R_{i}}\left\|\partial_{\ell_{1}} \ldots \partial_{\ell_{i}} u\right\|_{\left(L^{2}\right)_{s}}^{2}
$$

where $P^{k, i}$ is a finite subset (of indexes) of $\mathbb{R}^{i}$ that will be described below. Here and below we will use the iterated summation notation

$$
\sum_{\ell_{1}, \ell_{2}, \ldots, \ell_{k}}:=\sum_{\ell_{1} \in \mathbb{N}} \sum_{\ell_{2} \in \mathbb{N}} \cdots \sum_{\ell_{k} \in \mathbb{N}} .
$$

Similar result for the case $k=1$ and $k=2$ (with $s=0$ ) can be found in [11] and the corresponding spaces are denoted by $W^{1,2}(H, \mu)$ and $W^{2,2}(H, \mu)$ respectively. See Theorem 10.15 in page 147 and Equations (10.54)-(10.57) in page 162. Here, we generalize this results in [11] to any $k \in \mathbb{N}$ and $s<\lambda_{1}^{2 \theta} / 2$, see Theorem 12. Additionally, we introduce several intermediate results for general norms of derivatives which can be used for defining fractional derivatives. We note that Theorem 12 is a key tool for establishing the regularity theory in Section 4.4 .

We define the $k$-th derivative as follows. See $[11,24,28]$ and references therein.

Definition 5. For $k \in \mathbb{N}$ and $p \in \mathbb{R}$ define

$$
D^{k} u(\omega):=\sum_{\ell_{1}, \ell_{2}, \ldots, \ell_{k}} \partial_{\ell_{1}} \ldots \partial_{\ell_{k}} u(\omega) \eta_{\ell_{1}} \otimes \ldots \otimes \eta_{\ell_{k}} \in\left(\mathcal{S}^{\prime}\right)^{\otimes k}
$$

and with $\Gamma_{\oplus}(A)$ defined in (8) we set

$\Gamma_{\oplus}(A)^{\frac{p}{2}} D^{k} u(\omega):=\sum_{\ell_{1}, \ell_{2}, \ldots, \ell_{k}} \Gamma_{\oplus}(A)^{\frac{p}{2}} \partial_{\ell_{1}} \ldots \partial_{\ell_{k}} u(\omega) \eta_{\ell_{1}} \otimes \ldots \otimes \eta_{\ell_{k}} \in\left(\mathcal{S}^{\prime}\right)^{\otimes k}$

We also use the convention $D^{0} u=u$.

We will compute $\left(L_{2}\right)_{s}$-norms of derivatives according to the next definition.

Definition 6. For $k \in \mathbb{N}$ and $\boldsymbol{q}=\left(q_{1}, \ldots, q_{k}\right) \in \mathbb{R}^{k}$ define

$$
\begin{aligned}
\left\|D^{k} u\right\|_{\boldsymbol{q}}^{2} & =\left\|A^{q_{1}} \otimes \ldots \otimes A^{q_{k}} D^{k} u\right\|_{L^{2}\left(\mathcal{S}^{\prime},\left(L^{2}\right)_{s}^{\otimes k}\right)}^{2} \\
& =\sum_{\ell_{1}, \ell_{2}, \ldots, \ell_{k}} \lambda_{\ell_{1}}^{2 q_{1}} \ldots \lambda_{\ell_{k}}^{2 q_{k}}\left\|\partial_{\ell_{1}} \ldots \partial_{\ell_{k}} u\right\|_{\left(L^{2}\right)_{s}}^{2} .
\end{aligned}
$$

We also set $\left\|D^{0} u\right\|^{2}=\|u\|_{\left(L^{2}\right)_{s}}^{2}$. 
Now we prove some basic relations between derivatives in the $\omega$ variable and the operator $\Gamma_{\oplus}(A)$ defined in in (8). See [28] for related results.

Lemma 7. For all $p, q \in \mathbb{R}$ we have the following relations

$$
\begin{gathered}
\left(\Gamma_{\oplus}\left(A^{q}\right)+\lambda_{\ell}^{q}\right)^{\frac{p}{2}} \partial_{\ell} u=\partial_{\ell} \Gamma_{\oplus}\left(A^{q}\right)^{\frac{p}{2}} u, \\
\left(\Gamma_{\oplus}\left(A^{q}\right)+\lambda_{\ell_{1}}^{q}+\ldots+\lambda_{\ell_{k}}^{q}\right)^{\frac{p}{2}} \partial_{\ell_{1}} \partial_{\ell_{2}} \cdots \partial_{\ell_{k}} u=\partial_{\ell_{1}} \partial_{\ell_{2}} \cdots \partial_{\ell_{k}} \Gamma_{\oplus}\left(A^{q}\right)^{\frac{p}{2}} u, \\
\left(\Gamma_{\oplus}\left(A^{q}\right)+\left\langle\boldsymbol{\beta}, \boldsymbol{\lambda}^{q}\right\rangle\right)^{\frac{p}{2}} \partial^{\boldsymbol{\beta}} u=\partial^{\boldsymbol{\beta}} \Gamma_{\oplus}\left(A^{q}\right)^{\frac{p}{2}} u .
\end{gathered}
$$
and

Proof. Since $\partial_{\ell} u=\sum_{\boldsymbol{\alpha} \in \mathcal{J}} \alpha_{\ell} u_{\boldsymbol{\alpha}} H_{\boldsymbol{\sigma}(s)^{2}, \boldsymbol{\alpha}-\boldsymbol{\xi}_{\ell}}$, then

$$
\begin{aligned}
\left(\Gamma_{\oplus}\left(A^{q}\right)+\lambda_{\ell}^{q}\right)^{\frac{p}{2}} \partial_{\ell} u & =\sum_{\boldsymbol{\alpha} \in \mathcal{J}}\left(\left\langle\boldsymbol{\alpha}-\boldsymbol{\xi}_{\ell}, \boldsymbol{\lambda}^{q}\right\rangle+\lambda_{\ell}^{q}\right)^{\frac{p}{2}} \alpha_{\ell} u_{\boldsymbol{\alpha}} H_{\boldsymbol{\sigma}^{2}, \boldsymbol{\alpha}-\boldsymbol{\xi}_{\ell}} \\
& =\sum_{\boldsymbol{\alpha} \in \mathcal{J}}\left(\left\langle\boldsymbol{\alpha}, \boldsymbol{\lambda}^{q}\right\rangle-\lambda_{\ell}^{q}+\lambda_{\ell}^{q}\right)^{\frac{p}{2}} \alpha_{\ell} u_{\boldsymbol{\alpha}} H_{\boldsymbol{\sigma}^{2}, \boldsymbol{\alpha}-\boldsymbol{\xi}_{\ell}} \\
& =\sum_{\boldsymbol{\alpha} \in \mathcal{J}}\left\langle\boldsymbol{\alpha}, \boldsymbol{\lambda}^{q}\right\rangle^{\frac{p}{2}} \alpha_{\ell} u_{\boldsymbol{\alpha}} H_{\boldsymbol{\sigma}^{2}, \boldsymbol{\alpha}-\boldsymbol{\xi}_{\ell}}=\partial_{\ell} \Gamma_{\oplus}\left(A^{q}\right)^{\frac{1}{2}} u
\end{aligned}
$$

which prove (17). Note that (18) follows easily from (17) and (19) is consequence of (18) and the notation $\left\langle\boldsymbol{\beta}, \boldsymbol{\lambda}^{q}\right\rangle=\sum_{j=1}^{d(\boldsymbol{\alpha})} \beta_{j} \lambda_{j}^{q}$.

Lemma 8. For $k \in \mathbb{N}$ and $\boldsymbol{q} \in \mathbb{R}^{k}$ we have

$$
\begin{gathered}
\sum_{\ell_{k}} \lambda_{\ell_{k}}^{2 q_{k}}\left\|D^{k-1} \partial_{\ell_{k}} u\right\|_{\left(q_{1}, \ldots, q_{k-1}\right)}^{2}=\left\|D^{k} u\right\|_{\left(q_{1}, \ldots, q_{k}\right)}^{2}, \\
\left\|D \Gamma_{\oplus}\left(A^{2 q_{2}}\right)^{\frac{1}{2}} u\right\|_{q_{1}}^{2}=\left\|\Gamma_{\oplus}\left(A^{2 q_{2}}\right)^{\frac{1}{2}} D u\right\|_{q_{1}}^{2}+\|D u\|_{q_{1}+q_{2}}^{2}
\end{gathered}
$$

and for $\boldsymbol{q}=\left(q_{1}, \ldots, q_{k}\right) \in \mathbb{R}^{k}$ and $t \in \mathbb{R}$ we have

$$
\left\|D^{k} \Gamma_{\oplus}\left(A^{2 t}\right)^{\frac{1}{2}} u\right\|_{\boldsymbol{q}}^{2}=\left\|\Gamma_{\oplus}\left(A^{2 t}\right)^{\frac{1}{2}} D^{k} u\right\|_{\boldsymbol{q}}^{2}+\sum_{i=1}^{k}\left\|D^{k} u\right\|_{\boldsymbol{q}+t \boldsymbol{\xi}_{i}}^{2}
$$

where $\boldsymbol{q}+t \boldsymbol{\xi}_{i}=\left(q_{1}, \ldots, q_{i}+t, \ldots, q_{k}\right)$.

Proof. Equation (20) follows directly from Definition 6. We prove (21). Using Definitions 5 and 6 together with Equation (17),

$$
\begin{aligned}
\left\|D \Gamma_{\oplus}\left(A^{2 q_{2}}\right)^{\frac{1}{2}} u\right\|_{q_{1}}^{2} & =\sum_{\ell=1} \lambda_{\ell}^{2 q_{1}}\left\|\partial_{\ell} \Gamma_{\oplus}\left(A^{2 q_{2}}\right)^{\frac{1}{2}} u\right\|_{\left(L^{2}\right)_{s}}^{2} \\
& =\sum_{\ell=1} \lambda_{\ell}^{2 q_{1}}\left\|\left(\Gamma_{\oplus}\left(A^{2 q_{2}}\right)+\lambda_{\ell}^{2 q_{2}}\right)^{\frac{1}{2}} \partial_{\ell} u\right\|_{\left(L^{2}\right)_{s}}^{2} \\
& =\sum_{\ell=1} \lambda_{\ell}^{2 q_{1}}\left(\left\|\Gamma_{\oplus}\left(A^{2 q_{2}}\right)^{\frac{1}{2}} \partial_{\ell} u\right\|_{\left(L^{2}\right)_{s}}^{2}+\lambda_{\ell}^{2 q_{2}}\left\|\partial_{\ell} u\right\|_{\left(L^{2}\right)_{s}}^{2}\right) \\
& =\left\|\Gamma_{\oplus}\left(A^{2 q_{2}}\right)^{\frac{1}{2}} D u\right\|_{q_{1}}^{2}+\|D u\|_{q_{2}+q_{1}}^{2} .
\end{aligned}
$$


To prove (22) observe that using (18) we get

$$
\begin{aligned}
\left\|D^{k} \Gamma_{\oplus}\left(A^{2 t}\right)^{\frac{1}{2}} u\right\|_{\boldsymbol{q}}^{2}=\sum_{\ell_{1}, \ldots, \ell_{k}} \lambda_{\ell_{1}}^{2 q_{1}} \cdots \lambda_{\ell_{k}}^{2 q_{k}}\left\|\partial_{\ell_{1}} \cdots \partial_{\ell_{k}} \Gamma_{\oplus}\left(A^{2 t}\right)^{\frac{1}{2}} u\right\|_{\left(L^{2}\right)_{s}}^{2} \\
=\sum_{\ell_{1}, \ldots, \ell_{k}} \lambda_{\ell_{1}}^{2 q_{1}} \cdots \lambda_{\ell_{k}}^{2 q_{k}}\left\|\left(\Gamma_{\oplus}\left(A^{2 t}\right)+\lambda_{\ell_{1}}^{2 t}+\cdots+\lambda_{\ell_{k}}^{2 t}\right)^{\frac{1}{2}} \partial_{\ell_{1}} \cdots \partial_{\ell_{k}} u\right\|_{\left(L^{2}\right)_{s}}^{2} \\
=\sum_{\ell_{1}, \ldots, \ell_{k}} \lambda_{\ell_{1}}^{2 q_{1}} \cdots \lambda_{\ell_{k}}^{2 q_{k}}\left\|\Gamma_{\oplus}\left(A^{2 t}\right)^{\frac{1}{2}} \partial_{\ell_{1}} \cdots \partial_{\ell_{k}} u\right\|_{\left(L^{2}\right)_{s}}^{2} \\
\quad+\sum_{\ell_{1}, \ldots, \ell_{k}} \lambda_{\ell_{1}}^{2 q_{1}} \cdots \lambda_{\ell_{k}}^{2 q_{k}}\left(\lambda_{\ell_{1}}^{2 t}+\cdots+\lambda_{\ell_{k}}^{2 t}\right)\left\|\partial_{\ell_{1}} \cdots \partial_{\ell_{k}} u\right\|_{\left(L^{2}\right)_{s}}^{2} \\
=\left\|\Gamma_{\oplus}\left(A^{2 t}\right)^{\frac{1}{2}} D^{k} u\right\|_{\boldsymbol{q}}^{2}+\sum_{i=1}^{k}\left\|D^{k} u\right\|_{\boldsymbol{q}+t \boldsymbol{\xi}_{i}}^{2} .
\end{aligned}
$$

The following result reveals the basic relation between norms of derivatives and the norm $\|u\|_{p ; \rho, s}^{2}$ defined in (5) with weights in (7) for the values $p=1 / 2$ and $p=1$. This result will be used as the initial induction step in the proof of the equivalence of norms for any value of $p$ half a positive integer; see Theorem 12 .

Theorem 9. For any $k \in \mathbb{N}$ and $\boldsymbol{q}=\left(q_{1}, \ldots q_{k}\right) \in \mathbb{R}^{k}$ we have

$$
\begin{gathered}
\left\|\Gamma_{\oplus}\left(A^{2 q_{1}}\right)^{\frac{1}{2}} u\right\|_{\left(L^{2}\right)_{s}}^{2}=\sum_{\ell=1}^{\infty} \lambda_{\ell}^{2 q_{1}}\left\|\partial_{\ell} u\right\|^{2}=\|D u\|_{q_{1}}^{2}, \\
\left\|\Gamma_{\oplus}\left(A^{2 q_{k}}\right)^{\frac{1}{2}} D^{k-1} u\right\|_{\left(q_{1}, \ldots, q_{k-1}\right)}^{2}=\left\|D^{k} u\right\|_{\left(q_{1}, q_{2}, \ldots, q_{k}\right)}^{2}
\end{gathered}
$$

and we have the identities

$$
\begin{aligned}
\left\|\Gamma_{\oplus}\left(A^{2 q_{1}}\right)^{\frac{1}{2}} \Gamma_{\oplus}\left(A^{2 q_{2}}\right)^{\frac{1}{2}} u\right\|_{\left(L^{2}\right)_{s}}^{2} & =\left\|D^{2} u\right\|_{\left(q_{1}, q_{2}\right)}^{2}+\left\|\Gamma_{\oplus}\left(A^{2\left(q_{1}+q_{2}\right)}\right)^{\frac{1}{2}} u\right\|_{\left(L^{2}\right)_{s}}^{2} \\
& =\left\|D^{2} u\right\|_{\left(q_{1}, q_{2}\right)}^{2}+\|D u\|_{q_{1}+q_{2}}^{2} .
\end{aligned}
$$

and

$$
\begin{aligned}
& \left\|\Gamma_{\oplus}\left(A^{2 q_{1}}\right)^{\frac{1}{2}} \Gamma_{\oplus}\left(A^{2 q_{2}}\right)^{\frac{1}{2}} \Gamma_{\oplus}\left(A^{2 q_{3}}\right)^{\frac{1}{2}} u\right\|_{\left(L^{2}\right)_{s}}^{2}=\left\|D^{3} u\right\|_{\left(q_{1}, q_{2}, q_{3}\right)}^{2} \\
& +\left\|D^{2} u\right\|_{\left(q_{1}+q_{3}, q_{2}\right)}^{2}+\left\|D^{2} u\right\|_{\left(q_{1}, q_{2}+q_{3}\right)}^{2}+\left\|D^{2} u\right\|_{\left(q_{1}+q_{2}, q_{3}\right)}^{2} \\
& +\|D u\|_{\left(q_{1}+q_{2}+q_{3}\right)}^{2}
\end{aligned}
$$

Proof. From Equations (10) and (11) we have that

$$
\|D u\|_{q_{1}}^{2}=\sum_{\ell=1}^{\infty} \lambda_{\ell}^{2 q_{1}}\left\|\partial_{\ell} u\right\|_{\left(L^{2}\right)_{s}}^{2}=\sum_{\ell=1}^{\infty} \sum_{\alpha_{\ell} \geq 1} \alpha_{\ell} \lambda_{\ell}^{2 q_{1}} u_{\boldsymbol{\alpha}}^{2} \sigma^{2 \boldsymbol{\alpha}} \boldsymbol{\alpha} !
$$




$$
\begin{aligned}
& =\sum_{\boldsymbol{\alpha} \in \mathcal{J}}\left(\sum_{\ell=1}^{d(\boldsymbol{\alpha})} \alpha_{\ell} \lambda_{\ell}^{2 q_{1}}\right) u_{\boldsymbol{\alpha}}^{2} \boldsymbol{\sigma}^{2 \boldsymbol{\alpha}} \boldsymbol{\alpha} ! \\
& =\sum_{\boldsymbol{\alpha} \in \mathcal{J}}\left\langle\boldsymbol{\alpha}, \boldsymbol{\lambda}^{2 q_{1}}\right\rangle u_{\boldsymbol{\alpha}}^{2} \boldsymbol{\sigma}^{2 \boldsymbol{\alpha}} \boldsymbol{\alpha} !=\left\|\Gamma_{\oplus}\left(A^{2 q_{1}}\right)^{\frac{1}{2}} u\right\|_{\left(L^{2}\right)_{s}}^{2},
\end{aligned}
$$

and hence (23) holds. To prove (24) observe that from (23) and (20) we get

$$
\begin{aligned}
\| \Gamma_{\oplus}\left(A^{2 q_{k}}\right)^{\frac{1}{2}} & D^{k-1} u \|_{\left(q_{1}, \ldots, q_{k-1}\right)}^{2} \\
& =\sum_{\ell_{1}, \ldots, \ell_{k-1}} \lambda_{\ell_{1}}^{2 q_{1}} \ldots \lambda_{\ell_{k-1}}^{2 q_{k-1}}\left\|\Gamma_{\oplus}\left(A^{2 q_{k}}\right)^{\frac{1}{2}} \partial_{\ell_{1}} \ldots \partial_{\ell_{k-1}} u\right\|_{\left(L^{2}\right) s}^{2} \\
& =\sum_{\ell_{1}, \ldots, \ell_{k}} \lambda_{\ell_{1}}^{2 q_{1}} \ldots \lambda_{\ell_{k}}^{2 q_{k}}\left\|\partial_{\ell_{1}} \ldots \partial_{\ell_{k}} u\right\|_{\left(L^{2}\right) s}^{2}=\left\|D^{k} u\right\|_{\left(q_{1}, \ldots, q_{k}\right)}^{2} .
\end{aligned}
$$

To prove (25) observe that from (23), (21) and (24) we have

$$
\begin{aligned}
\left\|\Gamma_{\oplus}\left(A^{2 q_{1}}\right)^{\frac{1}{2}} \Gamma_{\oplus}\left(A^{2 q_{2}}\right)^{\frac{1}{2}} u\right\|_{\left(L^{2}\right)_{s}}^{2} & =\left\|D \Gamma_{\oplus}\left(A^{2 q_{2}}\right)^{\frac{1}{2}} u\right\|_{q_{1}}^{2} \\
& =\left\|\Gamma_{\oplus}\left(A^{2 q_{2}}\right)^{\frac{1}{2}} D u\right\|_{q_{1}}^{2}+\|D u\|_{q_{1}+q_{2}}^{2} \\
& =\left\|D^{2} u\right\|_{\left(q_{1}, q_{2}\right)}^{2}+\left\|\Gamma_{\oplus}\left(A^{2\left(q_{1}+q_{2}\right)}\right)^{\frac{1}{2}} u\right\|_{\left(L^{2}\right)_{s}}^{2} .
\end{aligned}
$$

For the proof of (26), see Theorem 12 where we prove the general case.

In order to write down the general version of formula (25) we shall introduce some notation. Consider the set of indexes $\{1,2, \ldots, k\}$ and its set of partitions $P^{k}$; see Charalambides [8]. Recall that, given $i \in \mathbb{N}$, an $i$-partition of $\{1,2, \ldots, k\}$ is a decomposition of this set into $i$ nonempty and disjoint subsets. We denote by $P^{k, i}$ the set of all $i$-partitions of $\{1,2, \ldots, k\}$. It is well known that $\#\left(P^{k, i}\right)=S(k, i)$, the Stirling number of the second kind (which is also the number of distributions of $k$ distinguishable balls into $i$ indistinguishable urns). Let each $i$-partition $R=\left(R_{1}, \ldots, R_{i}\right) \in P^{k, i}$, be ordered in such a way that

$$
\min R_{1}<\min R_{2}<\ldots<\min R_{i} .
$$

To each $i$-partition and $\boldsymbol{q}=\left(q_{1}, \ldots, q_{k}\right) \in \mathbb{R}^{k}$ we associate a multi-index $R(\boldsymbol{q})=$ $\left(R_{1}(\boldsymbol{q}), \ldots, R_{i}(\boldsymbol{q})\right) \in \mathbb{R}^{i}$ defined by

$$
R_{i^{\prime}}(\boldsymbol{q})=\sum_{i^{\prime \prime} \in R_{i^{\prime}}} q_{i^{\prime \prime}}, \quad i^{\prime}=1, \ldots, i .
$$

Example 10. Let $\boldsymbol{q}=\left(q_{1}, q_{2}, q_{3}\right)$ and consider the 2-partition $R=\left\{R_{1}=\right.$ $\left.\{1\}, R_{2}=\{2,3\}\right\}$. Then $R(\boldsymbol{q})=\left(q_{1}, q_{2}+q_{3}\right)$.

Example 11. Let $\boldsymbol{q}=(q, q, q, q)$ and consider the 3-partition $R=\left\{R_{1}=\right.$ $\left.\{1\}, R_{2}=\{2,3\}, R_{3}=\{4\}\right\}$. Then $R(\boldsymbol{q})=(q, 2 q, q)$. 
The following result gives a closed formula that allows us to compute the norm $\|\cdot\|_{p ; \rho, s}^{2}$ using $\omega$-partial derivatives. It shows the equivalence between the weighted chaos norms, using the weight (7), and the Gaussian Sobolev norms, defined using $\left(L^{2}\right)_{s}$ norms of derivatives.

Theorem 12. Let $k \in \mathbb{N}$ and $\boldsymbol{q}=\left(q_{1}, q_{2}, \ldots, q_{k}\right) \in \mathbb{R}^{k}$. We have

$$
\left\|\Gamma_{\oplus}\left(A^{2 q_{1}}\right)^{\frac{1}{2}} \ldots \Gamma_{\oplus}\left(A^{2 q_{k}}\right)^{\frac{1}{2}} u\right\|_{\left(L^{2}\right)_{s}}^{2}=\sum_{i=1}^{k} \sum_{R \in P^{k}, i}\left\|D^{i} u\right\|_{R(\boldsymbol{q})}^{2}
$$

In particular, if we take $\boldsymbol{q}=\frac{1}{2} \mathbf{1}_{k}$ where $\mathbf{1}_{k}:=(1, \ldots, 1) \in \mathbb{N}^{k}$

$$
\left\|\Gamma_{\oplus}(A)^{p} u\right\|_{\left(L^{2}\right)_{s}}^{2}=\sum_{i=1}^{k} \sum_{R \in P^{k, i}}\left\|D^{i} u\right\|_{R\left(\frac{1}{2} \mathbf{1}_{k}\right)}^{2}
$$

and

$$
\|u\|_{\frac{k}{2} ; \rho, s}^{2}=\|u\|_{\left(L^{2}\right)_{s}}^{2}+\left\|\Gamma_{\oplus}(A)^{p} u\right\|_{\left(L^{2}\right)_{s}}^{2}=\|u\|_{\left(L^{2}\right)_{s}}^{2}+\sum_{i=1}^{k} \sum_{R \in P^{k}, i}\left\|D^{i} u\right\|_{R\left(\frac{1}{2} \mathbf{1}_{k}\right)}^{2} .
$$

Proof. We proceed by induction on $k$. For $k=1$ and $k=2$ we already proved the result, see (23) and (25) of Theorem 9.

Assume that (27) is valid for the first $k \in \mathbb{N}$. Then we have

$$
\begin{aligned}
& \left\|\Gamma_{\oplus}\left(A^{2 q_{1}}\right)^{\frac{1}{2}} \ldots \Gamma_{\oplus}\left(A^{2 q_{k+1}}\right)^{\frac{1}{2}} u\right\|_{\left(L^{2}\right)_{s}}^{2} \\
& \quad=\sum_{i=1}^{k} \sum_{R \in P^{(i)}}\left\|D^{i} \Gamma_{\oplus}\left(A^{2 q_{k+1}}\right)^{\frac{1}{2}} u\right\|_{R(\boldsymbol{q})}^{2} \\
& \quad=\sum_{i=1}^{k} \sum_{R \in P^{(i)}}\left(\left\|\Gamma_{\oplus}\left(A^{2 q_{k+1}}\right)^{\frac{1}{2}} D^{i} u\right\|_{R\left(q_{1}, \ldots, q_{k}\right)}^{2}+\sum_{i^{\prime}=1}^{i}\left\|D^{i} u\right\|_{R\left(q_{1}, \ldots, q_{k}\right)+q_{k+1} \boldsymbol{\xi}_{i^{\prime}}}^{2}\right)
\end{aligned}
$$

where we have used formula (22). Then, from (24) we get

$$
\begin{aligned}
& \left\|\Gamma_{\oplus}\left(A^{2 q_{1}}\right)^{\frac{1}{2}} \ldots \Gamma_{\oplus}\left(A^{2 q_{k+1}}\right)^{\frac{1}{2}} u\right\|_{\left(L^{2}\right)_{s}}^{2} \\
& \quad=\sum_{i=1}^{k} \sum_{R \in P^{k, i}}\left(\left\|D^{i} u\right\|_{\left(R\left(q_{1}, \ldots, q_{k}\right), q_{k+1}\right)}^{2}+\sum_{i^{\prime}=1}^{i}\left\|D^{i} u\right\|_{R\left(q_{1}, \ldots, q_{k}\right)+q_{k+1} \boldsymbol{\xi}_{i^{\prime}}}^{2}\right) \\
& \quad=\sum_{i=1}^{k+1} \sum_{R \in P^{k+1, i}}\left\|D^{i} u\right\|_{R\left(q_{1}, \ldots, q_{k+1}\right)}^{2} .
\end{aligned}
$$

To obtain the last equality we observe that the $i$-partitions $P^{k+1, i}$ of the set $\{1, \ldots, k+1\}$ are of the form $\{R,\{k+1\}\}$ where $R \in P^{k, i-1}$ or $R=\left(R_{1}, \ldots, R_{i^{\prime}} \cup\right.$ $\left.\{k+1\}, \ldots, R_{i}\right)$ for $1 \leq i^{\prime} \leq i$ and $R \in P^{k, i}$. 
Remark 13. Note that, given $r=\left(r_{\ell}\right)_{\ell=1}^{i} \in \mathbb{N}^{i}$, (see [8])

$$
\#\left(\left\{R \in P^{k, i}: \frac{1}{2} r=R\left(\frac{1}{2} \mathbf{1}_{k}\right)\right\}=\prod_{j=1}^{i-1}\left(\begin{array}{c}
\sum_{\ell=j}^{i} r_{\ell}-1 \\
r_{j}-1
\end{array}\right) .\right.
$$

\subsubsection{A remark on Kondratiev type norms}

In this section we study another classical weighted norm. We select different weights in the general norm defined in (5). Given a multi-index $\boldsymbol{\alpha}$ we denote $\lambda^{\boldsymbol{\alpha}}:=\prod_{j=1}^{d(\boldsymbol{\alpha})} \lambda_{j}^{\alpha_{j}}$. Take $\nu \in[0,1)$ and

$$
\rho(\boldsymbol{\alpha}, p)^{2}=(\boldsymbol{\alpha} !)^{\nu} \boldsymbol{\lambda}^{2 p \boldsymbol{\alpha}}, \quad \boldsymbol{\alpha} \in \mathcal{J}
$$

in (5). See $[18,20,26]$. Let us denote by \|\|$u \|_{p ; \rho, s}^{2}$ the resulting weighted norm. Note that we can write

$$
\|\| u\left\|\left.\right|_{p ; \rho, s} ^{2}=\right\| \Gamma_{\otimes, \nu}(A)^{p} u \|_{\left(L^{2}\right)_{s}}^{2}=\int_{\mathcal{S}^{\prime}}\left|\Gamma_{\otimes, \nu}(A)^{p} u(\omega)\right|^{2} e^{s\|\omega\|_{-\theta}^{2}} d \mu(\omega),
$$

where $\Gamma_{\otimes, \nu}(A)$ is the operator defined by $\Gamma_{\otimes, \nu}(A) H_{\boldsymbol{\sigma}^{2}, \boldsymbol{\alpha}}=(\alpha !)^{\nu} \boldsymbol{\lambda}^{\boldsymbol{\alpha}} H_{\boldsymbol{\sigma}^{2}, \boldsymbol{\alpha}}$. Note also that $\Gamma_{\otimes, 0}\left(A^{p}\right)=\Gamma_{\otimes, 0}(A)^{p}$. In the case of $\nu=0$ and $s=0, \Gamma_{\otimes, 0}(A)$ is called the Second Quantization of $A$; see [17].

For a priori error estimates for Lemma 19 using ||$\cdot \mid \|_{p ; \rho, s}^{2}$, we refer to [14]. Now we show how to compute the norm $\|\mid \cdot\|_{p ; \rho, s}$ defined above for the case $\nu=0$. We use the notation $\left(\boldsymbol{\lambda}^{p}-\mathbf{1}\right)^{\boldsymbol{\gamma}}=\prod_{j=1}^{d(\boldsymbol{\gamma})}\left(\lambda_{j}^{p}-1\right)^{\gamma_{j}}$. Recall that $1<\lambda_{1} \leq$ $\lambda_{2} \leq \ldots$. We have

$$
\begin{aligned}
\lambda^{2 p \boldsymbol{\alpha}} & =\prod_{j=1}^{d(\boldsymbol{\alpha})}\left(\lambda_{j}^{2 p}-1+1\right)^{\alpha_{j}}=\prod_{j=1}^{d(\boldsymbol{\alpha})}\left(\sum_{\gamma_{j} \leq \alpha_{j}}\left(\begin{array}{c}
\alpha_{j} \\
\gamma_{j}
\end{array}\right)\left(\lambda_{j}^{2 p}-1\right)^{\gamma_{j}}\right) \\
& =\sum_{\boldsymbol{\gamma} \leq \boldsymbol{\alpha}}\left(\begin{array}{l}
\boldsymbol{\alpha} \\
\gamma
\end{array}\right)\left(\boldsymbol{\lambda}^{2 p}-\mathbf{1}\right)^{\gamma} .
\end{aligned}
$$

Then

$$
\begin{aligned}
\sum_{\boldsymbol{\gamma} \in \mathcal{J}} \frac{\left(\boldsymbol{\lambda}^{2 p}-\mathbf{1}\right)^{\gamma}}{\gamma !}\left\|\partial^{\gamma} u\right\|_{\left(L^{2}\right)_{s}}^{2} & =\sum_{\boldsymbol{\gamma} \in \mathcal{J}} \frac{\left(\boldsymbol{\lambda}^{2 p}-\mathbf{1}\right)^{\gamma}}{\gamma !} \sum_{\boldsymbol{\alpha} \geq \gamma} \frac{\boldsymbol{\alpha} !}{(\boldsymbol{\alpha}-\gamma) !} u_{\boldsymbol{\alpha}}^{2} \boldsymbol{\sigma}^{2 \boldsymbol{\alpha}} \boldsymbol{\alpha} ! \\
& =\sum_{\boldsymbol{\alpha} \in \mathcal{J}}\left(\sum_{\gamma \leq \boldsymbol{\alpha}} \frac{\boldsymbol{\alpha} !}{\gamma !(\boldsymbol{\alpha}-\gamma) !}\left(\boldsymbol{\lambda}^{2 p}-\mathbf{1}\right)^{\gamma}\right) u_{\boldsymbol{\alpha}}^{2} \boldsymbol{\sigma}^{2 \boldsymbol{\alpha}} \boldsymbol{\alpha} ! \\
& =\sum_{\boldsymbol{\alpha} \in \mathcal{J}} \boldsymbol{\lambda}^{2 p \boldsymbol{\alpha}} u_{\boldsymbol{\alpha}}^{2} \boldsymbol{\sigma}^{2 \boldsymbol{\alpha}} \boldsymbol{\alpha} !=\|u\| \|_{p ; \rho, s}^{2} .
\end{aligned}
$$

Summarizing we have \|\|$u\left\|_{p ; \rho, s}^{2}=\sum_{\boldsymbol{\gamma} \in \mathcal{J}} \frac{\left(\boldsymbol{\lambda}^{2 p}-\mathbf{1}\right)^{\gamma}}{\boldsymbol{\gamma} !}\right\| \partial^{\gamma} u \|_{\left(L^{2}\right)_{s}}^{2}$. We conclude that in order to have \|\|$u \|_{p ; \rho, s}<\infty$ we need all partial derivative of all orders 
being $\left(L^{2}\right)_{s}$ functions with the series above being finite. Then, in oder to obtain regularity results using partial derivative in the $\omega$ variable, we have to bound all partial derivatives $\partial^{\gamma} u$ of all orders of the solution of (30). Moreover, we need a bound for the weighted sum $\sum_{\boldsymbol{\gamma} \in \mathcal{J}} \frac{\left(\boldsymbol{\lambda}^{2 p}-\mathbf{1}\right)^{\boldsymbol{\gamma}}}{\boldsymbol{\gamma} !}\left\|\partial^{\gamma} u\right\|_{\left(L^{2}\right)_{s}}^{2}$. Due to this technical difficulties, we consider only the norm $\|u\|_{p ; \rho, s}^{2}$ (introduced in Section $3.2)$ to analyze and measure the stochastic regularity of the solution of the stochastic pressure equation. We recall that, from Theorem 12, in order to obtain regularity results in the norm $\|u\|_{p ; \rho, s}^{2}$ (defined in Section 3.2) we only need to bound a finite number of partial derivatives, see (16).

\section{Application to elliptic equations with lognor- mal coefficients}

\subsection{Examples and comments}

In this section we show a way to deal with nonuniform ellipticity in the case of one dimensional log-normal noise. In the next section we present a summary of the extension of this argument to the case of infinity-dimensional log-normal noise. We consider the following toy problem,

$$
-\left(e^{\xi_{1} a_{1}(x)} u_{x}\right)_{x}=f(x), \text { in }(0,1)
$$

with $u(0)=u(1)=0$. Here the right hand side is deterministic and $\xi_{1}$ has normal distribution with mean zero and variance 1 . We observe that, for any $\epsilon>0$ we have

$$
e^{-\frac{C_{1}}{2 \epsilon}} e^{-\frac{\epsilon}{2} \xi_{1}^{2}} \leq e^{\xi_{1} a_{1}(x)} \leq e^{-\frac{C_{1}}{2 \epsilon}} e^{\frac{\epsilon}{2} \xi_{1}^{2}}
$$

where $C_{1}=\max _{x \in \mathcal{O}} a_{1}(x)$. Then we obtain ellipticity and boundedness of the log-normal coefficient in terms of quantities involving random variables that are easy to handle. The idea is then to use weights of the type $e^{s \xi^{2}}$ into the norms used for the analysis. The factor $e^{s \xi^{2}}$ is it easy to handle in computations. Since $\xi$ is normal with unit variance, then $\int G(\xi) e^{s \xi^{2}} d \xi=\frac{1}{\sqrt{2 \pi}} \int_{-\infty}^{\infty} G(r) e^{s r^{2}-\frac{1}{2} r^{2}} d r$. Using the Lax-Milgram lemma for each $\xi$ and integrating in $\xi$, we obtain (for $s$ and $\epsilon>0$ such that $s+\epsilon<1 / 2$ )

$$
\begin{aligned}
\int\left|u_{x}(x, \xi)\right|^{2} e^{s \xi^{2}} d \xi & =\frac{1}{\sqrt{2 \pi}} \int_{-\infty}^{\infty} \int_{0}^{1}\left|u_{x}(x, r)\right|^{2} d x e^{s r^{2}-\frac{1}{2} r^{2}} d r \\
& \leq C e^{\frac{C_{1}}{2 \epsilon}}\|f\|_{H^{-1}(0,1)}^{2} \frac{1}{\sqrt{2 \pi}} \int_{-\infty}^{\infty} e^{(s+\epsilon) r^{2}-\frac{1}{2} r^{2}} d r \\
& =C e^{\frac{C_{1}}{2 \epsilon}}(1-2(s+\epsilon))^{-\frac{1}{2}}\|f\|_{H^{-1}(0,1)}^{2}
\end{aligned}
$$

Observe that, taking $s=0$ and $\epsilon<1 / 2, u_{x} \in L^{2}(\mathcal{O}) \otimes L^{2}(d \xi)$. Moreover,

$$
\frac{1}{\sqrt{2 \pi}} \int_{-\infty}^{\infty}\left|u_{x}\right|^{2} e^{-\frac{1}{2} r^{2}} d x d r \leq C e^{\frac{C_{1}}{2 \epsilon}}(1-2 \epsilon)^{-\frac{1}{2}}\|f\|_{H^{-1}(0,1)}^{2}
$$


Having solutions in tensor product (of Hilbert) spaces is convenient to device approximation procedures, see [14]. The introduction of the Hilbert norms with weight $e^{s \xi^{2}}$ with $s \neq 0$ is convenient in case the right hand side is random and has extra (or lack some) decay in terms of $\xi$. In [14] we extended the argument above to the case of infinite dimensional lognormal noise. In this work, we showed that the White Noise Analysis is a very convenient framework for this task. The results in [14] are independent of the White Noise Analysis framework and can be stated using other convenient infinity dimensional calculus framework.

\subsection{Dealing with non-uniform ellipticity using a white noise approach}

In this section we summarize the procedure introduced in [14] to deal with non-uniform ellipticity in the case of infinity dimensional log-normal noise. For further details we refer to [14]. Some of these ideas were extended to other random coefficients in [25]. Some of the results presented here will be used in the derivation of regularity results for problem (30). We use the notation and White Noise Framework introduced in Section 2.

First, we summarize the model problem used in [14]. We need the following definition.

Definition 14. The 1-dimensional smoothed White Noise associated to $H$ and $A$ is the map $w: \mathcal{S} \times \mathcal{S}^{\prime} \longrightarrow \mathbb{R}$ given by $w(\xi)=w(\xi, \omega)=\langle\omega, \xi\rangle$ for $\omega \in \mathcal{S}^{\prime}$, $\xi \in \mathcal{S}$. Let $\mathcal{O} \subset \mathbb{R}^{d}$. Using the 1-dimensional smoothed White Noise $w$ we can construct a stochastic process, called the smoothed White Noise process $W_{\phi}(x, \omega)$, as follows:

$$
W_{\phi}(x, \omega):=w\left(\phi_{x}, \omega\right)=\left\langle\omega, \phi_{x}\right\rangle, x \in \mathcal{O}, \omega \in \mathcal{S}^{\prime},
$$

where $\phi_{x} \in H$ for all $x \in \mathcal{O}$. For each $x \in \mathcal{O}, W_{\phi}(x, \cdot)$ is normally distributed with zero mean and for $x, \hat{x} \in \mathcal{O}$ we can write

$$
W_{\phi}(x, \omega)=\sum_{j=1}^{\infty}\left(\eta_{j}, \phi_{x}\right)_{H}\left\langle\omega, \eta_{j}\right\rangle
$$

where the $\left\langle\omega, \eta_{j}\right\rangle$ are independent and identically standard normal distributions and it is easy to see that $E_{\mu} W_{\phi}(x, \cdot) W_{\phi}(\hat{x}, \cdot)=\left(\phi_{x}, \phi_{\hat{x}}\right)_{H}$.

From now on we assume that $\mathcal{O}$ is an open connected subdomain of $\mathbb{R}^{d}$ with Liptschitz boundary. Given $\phi_{x} \in \mathcal{S}_{\theta}$ for all $x \in \mathcal{O}$ we consider the following problem: For all $\omega \in \mathcal{S}^{\prime}$, find $u(x, \omega ; \phi)$ such that

$$
\left\{\begin{aligned}
-\nabla_{x} \cdot\left(\kappa(x, \omega ; \phi) \nabla_{x} u(x, \omega ; \phi)\right) & =f(x, \omega), \text { for all } x \in \mathcal{O} \\
u(x, \cdot ; \phi) & =0, \text { for all } x \in \partial \mathcal{O},
\end{aligned}\right.
$$

where

$$
\kappa(x, \omega ; \phi):=e^{W_{\phi}(x, \omega)}=e^{\left\langle\omega, \phi_{x}\right\rangle}
$$


and the exponent $W_{\phi}(x, \omega)$ is the 1-dimensional smoothed White Noise process of Definition 14. Thus, $\kappa$ is log-normal random process. Observe that for different maps $x \mapsto \phi_{x} \in \mathcal{S}_{\theta}$ there exists a different permeability function $\kappa(\cdot, \cdot, \phi)$ associated to it. We will omit, whenever there is no danger of confusion, the dependence of $\kappa$ on the map $x \mapsto \phi_{x}$ just to make the notation less cumbersome.

We have the following two important examples of construction of the mapping $x \mapsto \phi_{x}$. See Section 7 of [14] for more details.

Example 15. Let $\mathcal{O} \subset \mathbb{R}^{d}$ and take $H=L^{2}(\mathcal{O})$ and $A=Q^{-1}$, where $Q$ : $L^{2}(\mathcal{O}) \rightarrow L^{2}(\mathcal{O})$ is the integral operator on $(\mathcal{O} \times \mathcal{O})$ with kernel given by a covariance $C(x, \hat{x})$. In this case, for $x, \hat{x} \in \mathcal{O}$ we define $\phi_{x}(\hat{x})=\sum_{j=1}^{\infty} \lambda_{j}{ }^{-1 / 2} \eta_{j}(x) \eta_{j}(\hat{x})$, where $\lambda_{j}$ and $\eta_{j}$ are the eigenvalues and eigenfunctions of $A$. It is easy to see that $E_{\mu}\left(W_{\phi}(x, \cdot) W_{\phi}(\hat{x}, \cdot)\right)=C(x, \hat{x})$.

Example 16. We can take $H=L^{2}\left(\mathbb{R}^{d}\right)$ and $A=A_{1} \otimes \cdots \otimes A_{d}$ where $A_{i}=$ $-\frac{d^{2}}{d x^{2}}+x_{i}^{2}+1$. The eigenfunctions of $A_{i}$ are the $\ell$-th Hermite function with associated eigenvalue $2 \ell$, for all $\ell \in \mathbb{N}$. The $\eta_{j}$ and $\lambda_{j}$ are obtained by tensor product operations. Let $\phi_{x}(\hat{x})=\phi(\hat{x}-x), x \in \mathcal{O}$ and $\hat{x} \in \mathbb{R}^{d}$, where the window $\phi$ can be chosen such that the diameter of the support of $\phi$ is the maximum distance which $W_{\phi}(x, \cdot)$ and $W_{\phi}(\hat{x}, \cdot)$ might be correlated; see [18].

Following [14] we denote

$$
C_{\theta}=C_{\theta}(\phi):=\sup _{x \in \mathcal{O}}\left\|\phi_{x}\right\|_{\theta}
$$

Then we have for all $\epsilon>0$ and almost sure all $\omega \in \mathcal{S}^{\prime}$

$$
\kappa_{\min }(\omega):=e^{-\frac{C_{\theta}^{2}}{2 \epsilon}} e^{-\frac{\epsilon}{2}\|\omega\|_{-\theta}^{2}} \leq \kappa(x, \omega) \leq e^{\frac{C_{\theta}^{2}}{2 \epsilon}} e^{\frac{\epsilon}{2}\|\omega\|_{-\theta}^{2}}=: \kappa_{\max }(\omega) .
$$

Define $\mathcal{U}_{s}^{m}$ as the space of functions $u: \mathcal{O} \times \mathcal{S}^{\prime} \rightarrow \mathbb{R}$ such that

$$
\int_{\mathcal{S}^{\prime}}\|u(\cdot, \omega)\|_{H^{m}(\mathcal{O})}^{2} e^{s\|\omega\|_{-\theta}^{2}} d \mu(\omega)<+\infty
$$

with norm

$$
\|u\|_{\mathcal{U}_{s}^{m}}^{2}:=\int_{\mathcal{S}^{\prime}}\|u(\cdot, \omega)\|_{H^{m}(\mathcal{O})}^{2} e^{s\|\omega\|_{-\theta}^{2}} d \mu(\omega)
$$

and seminorm

$$
|u|_{\mathcal{U}_{s}^{m}}^{2}:=\int_{\mathcal{S}^{\prime}}|u(\cdot, \omega)|_{H^{m}(\mathcal{O})}^{2} e^{s\|\omega\|_{-\theta}^{2}} d \mu(\omega)
$$

Here we used the notation $H^{m}(\mathcal{O})$ to denote standard Sobolev spaces with seminorm $|h|_{H^{m}(\mathcal{O})}^{2}=\int_{\mathcal{O}} \sum_{|\boldsymbol{i}|=m}\left|\partial_{x}^{\boldsymbol{i}} h(x)\right|^{2} d x$ where $\boldsymbol{i}=\left(i_{1}, \ldots, i_{d}\right)$ and $\partial^{\boldsymbol{i}}$ denotes the partial derivative associated to the multiindex $i$. We also used the norm $\|h\|_{H^{m}(\mathcal{O})}^{2}=\|h\|_{L^{2}(\mathcal{O})}^{2}+\sum_{m^{\prime}=1}^{m}|h|_{H^{m^{\prime}}(\mathcal{O})}^{2}$

Note that $\mathcal{U}_{0}^{0}=L^{2}(\mathcal{O}) \otimes\left(L^{2}\right)$ and in general $\mathcal{U}_{s}^{m}=H^{m}(\mathcal{O}) \otimes\left(L^{2}\right)_{s}$ where $\left(L^{2}\right)_{s}$ was defined in $(3)$. We also define $\widehat{\mathcal{U}}_{s}^{1}=H_{0}^{1}(\mathcal{O}) \otimes\left(L^{2}\right)_{s} \subset \mathcal{U}_{s}^{1}$, i.e., the 
functions in $\mathcal{U}_{s}^{1}$ which vanish on $\partial \mathcal{O}$ almost sure in $\omega$. By using a Poincaré inequality, the seminorm $|\cdot|_{\mathcal{U}_{s}^{1}}$ is a norm equivalent to $\|\cdot\|_{\mathcal{U}_{s}^{1}}$ in $\widehat{\mathcal{U}}_{s}^{1}$. Since the space $\left(L^{2}\right)_{s}$ is the dual of $\left(L^{2}\right)_{-s}$ and the $H^{-1}(\mathcal{O})$ is the dual of $H_{0}^{1}(\mathcal{O})$, we can identify the dual space of $\widehat{\mathcal{U}}_{-s}^{1}$ with $\mathcal{U}_{s}^{-1}$.

We note that $\kappa(x, \omega)>0$ is neither uniformly bounded nor uniformly bounded away from zero, hence, the bilinear

$$
a(u, v)=\int_{\mathcal{O} \times \mathcal{S}^{\prime}} \kappa(x, \omega) \nabla u(x, \omega) \nabla v(x, \omega) d x d \mu
$$

is neither continuous nor coercive on $\widehat{\mathcal{U}}_{s}^{1} \times \widehat{\mathcal{U}}_{-s}^{1}$. According to [14], the coerciveness (the inf-sup condition) and boundedness of the bilinear form $a(\cdot, \cdot)$ can be circumvent by enlarging the space of test functions for $v$ from $\widehat{\mathcal{U}}_{-s}^{1}$ to $\widehat{\mathcal{U}}_{-s-\epsilon}^{1}$ and by reducing the solution space for $u$ from $\widehat{\mathcal{U}}_{s}^{1}$ to $\widehat{\mathcal{D}}_{s}^{1}$ where

$$
\widehat{\mathcal{D}}_{s}^{1}:=\left\{u \in \widehat{\mathcal{U}}_{s}^{1}: \sup _{v \in \widehat{\mathcal{U}}_{-s-\epsilon}^{1} \backslash\{0\}} \frac{a(u, v)}{|v|_{\mathcal{U}_{-s-\epsilon}^{1}}}<\infty\right\} .
$$

The weak formulation of problem (30) is then introduced as follows:

$$
\left\{\begin{array}{l}
\text { Given } f \in \mathcal{U}_{s+\epsilon}, \text { find } u \in \widehat{\mathcal{D}}_{s}^{1} \text { such that } \\
a(u, v)=\langle f, v\rangle \text { for all } v \in \widehat{\mathcal{U}}_{-s-\epsilon}^{1}
\end{array}\right.
$$

where the bilinear form $a$ is defined in (35) and the duality pairing between $f \in \mathcal{U}_{s+\epsilon}^{1}$ and $v \in \widehat{\mathcal{U}}_{-s-\epsilon}$ is given by $\langle f, v\rangle=\int_{\mathcal{O} \times \mathcal{S}^{\prime}} f(x, \omega) v(x, \omega) d x d \mu$.

Lemma 17 ([14] Existence and uniqueness of solutions). Let $\epsilon>0$ and assume that $C_{\theta}=\sup _{x \in \mathcal{O}}\left\|\phi_{x}\right\|_{\theta}<\infty$. Then for $f \in \mathcal{U}_{s+\epsilon}^{-1}$, there exists a unique solution $u \in \widehat{\mathcal{D}}_{s}^{1} \subset \widehat{\mathcal{U}}_{s}^{1}$ of Problem (36) and

$$
\|u\|_{\mathcal{U}_{s}^{1}} \leq C e^{\frac{C_{\theta}^{2}}{2 \epsilon}}\|f\|_{\mathcal{U}_{s+\epsilon}^{-1}}
$$

where $C=\sqrt{1+C_{\text {poin }}}$ and $C_{\text {poin }}$ is the Poincaré inequality constant which is independent of $\epsilon$ and $\theta$.

Remark 18. From Lemma 17, when $f \in \mathcal{U}_{0}^{-1}$ then for every $s<0$ (take $\epsilon=-s$ ) the solution $u \in \widehat{\mathcal{U}}_{s}^{1}$. In order to have $u \in \widehat{\mathcal{U}}_{0}^{1}$ we need $f \in \mathcal{U}_{\epsilon}^{-1}$ for some $\epsilon>0$. When the right-hand side $f$ is deterministic or is given by a finite sum of Fourier-Hermite polynomials, we have the solution $u \in \widehat{\mathcal{U}}_{s}^{1}$ for every $s$ satisfying $s<\frac{\lambda_{1}^{2 \theta}}{2} ;$ see Definition 2 and Theorem 3.

\subsection{Galerkin approximation and a priori error estimates using Weighted norms}

In this section we present an a priori error estimate obtained in [14]. The regularity results assumed in this error estimates are a main motivation for the studies developed in this paper. 

by

The corresponding tensor product norm for $u \in \mathcal{U}_{s}^{m}$ with $s<\frac{\lambda_{1}^{2 \theta}}{2}$ is given

$$
\|u\|_{\mathcal{U}_{s}^{m}}^{2}=\sum_{\boldsymbol{\alpha} \in \mathcal{J}} \boldsymbol{\alpha} ! \boldsymbol{\sigma}(s)^{2 \boldsymbol{\alpha}}\left\|u_{\boldsymbol{\alpha}, s}\right\|_{H^{m}(\mathcal{O})}^{2}
$$

where $u=\sum_{\boldsymbol{\alpha} \in \mathcal{J}} u_{\boldsymbol{\alpha}, s} H_{\boldsymbol{\sigma}(s)^{2}, \boldsymbol{\alpha}}$ with $u_{\boldsymbol{\alpha}, s} \in H^{m}(\mathcal{O})$ for all $\boldsymbol{\alpha} \in \mathcal{J}$.

Norms $\|\cdot\|_{p ; \rho, s}$ defined in (5) can also be extended to tensor products. The corresponding norms for the tensor product spaces $\mathcal{U}_{p ; \rho, s}^{m}:=H^{m}(\mathcal{O}) \otimes \mathcal{S}_{p, \rho, s}$ are defined by

$$
\|u\|_{\mathcal{U}_{p ; \rho, s}^{m}}^{2}=\sum_{\boldsymbol{\alpha} \in \mathcal{J}}\left(1+\langle\boldsymbol{\alpha}, \boldsymbol{\lambda}\rangle^{2 p}\right) \boldsymbol{\alpha} ! \boldsymbol{\sigma}(s)^{2 \boldsymbol{\alpha}}\left\|u_{\boldsymbol{\alpha}, s}\right\|_{H^{m}(\mathcal{O})}^{2},
$$

and we also introduce the seminorms,

$$
|u|_{\mathcal{U}_{p ; \rho, s}^{m}}^{2}:=\sum_{\boldsymbol{\alpha} \in \mathcal{J}}\left(1+\langle\boldsymbol{\alpha}, \boldsymbol{\lambda}\rangle^{2 p}\right) \boldsymbol{\alpha} ! \boldsymbol{\sigma}(s)^{2 \boldsymbol{\alpha}}\left|u_{\boldsymbol{\alpha}, s}\right|_{H^{m}(\mathcal{O})}^{2}
$$

Let $N, K \in \mathbb{N}_{0}$ and define

$$
\mathcal{J}^{N, K}=\{\boldsymbol{\alpha} \in \mathcal{J}: d(\boldsymbol{\alpha}) \leq K, \text { and },|\boldsymbol{\alpha}| \leq N\}
$$

and

$$
\mathcal{P}^{N, K}:=\operatorname{span}\left\{H_{\boldsymbol{\sigma}(s)^{2}, \boldsymbol{\alpha}}: \boldsymbol{\alpha} \in \mathcal{J}^{N, K}\right\}=\operatorname{span}\left\{\prod_{j=1}^{d(\boldsymbol{\alpha})}\left\langle\omega, \eta_{j}\right\rangle^{\alpha_{j}}: \boldsymbol{\alpha} \in \mathcal{J}^{N, K}\right\}
$$

i.e., $\mathcal{P}^{N, K}$ consists of polynomials in $\left\langle\omega, \eta_{1}\right\rangle, \ldots,\left\langle\omega, \eta_{K}\right\rangle$ of total degree at most $N$.

Note that when $s<\frac{\lambda_{1}^{2 \theta}}{2}$, polynomials in $\omega$ belong to $\left(L^{2}\right)_{s}$. Let $X_{0}^{h}(\mathcal{O}) \subset$ $H_{0}^{1}(\mathcal{O})$ be the finite element space of piecewise linear and continuous functions with respect to a quasi-uniform triangulation of $\mathcal{O}$ with mesh size $h$.

For $N, K \in \mathbb{N}_{0}$ and $h>0$ define the following discrete spaces:

$$
\mathcal{X}_{s}^{N, K, h}:=X_{0}^{h}(\mathcal{O}) \otimes \mathcal{P}^{N, K} \subset \widehat{\mathcal{U}}_{s}^{1} \subset \mathcal{U}_{s}^{1}
$$

and

$$
\mathcal{Y}_{s}^{N, K, h}:=\left\{v: v(x, \omega)=\tilde{v}(x, \omega) e^{\left(s+\frac{\epsilon}{2}\right)\left\|\Pi_{K} \omega\right\|_{-\theta}^{2}}, \tilde{v} \in \mathcal{X}_{s}^{N, K, h}\right\} \subset \widehat{\mathcal{U}}_{-(s+\epsilon)}^{1},
$$

where $\Pi_{K}$ is the $(H$-orthogonal $)$ projection on the $\operatorname{span}\left\{\eta_{1}, \ldots, \eta_{K}\right\}$ is defined by $\Pi_{K} \omega:=\sum_{j=1}^{K}\left\langle\omega, \eta_{j}\right\rangle \eta_{j}$, for all $\omega \in \mathcal{S}^{\prime}$. The discrete version of problem (36) is introduced as:

$$
\left\{\begin{array}{l}
\text { Find } u_{s}^{N, K, h} \in \mathcal{X}_{s}^{N, K, h} \text { such that } \\
a\left(u_{s}^{N, K, h}, v\right)=\langle f, v\rangle \text { for all } v \in \mathcal{Y}_{s}^{N, K, h} .
\end{array}\right.
$$

The corresponding discrete inf-sup condition, resulting linear system and one spatial dimension numerical example are discussed in [14].

We have the following a priori error estimates. See [14]. 
Lemma 19. Let $s \in \mathbb{R}$ and $u \in \widehat{\mathcal{U}}_{s}^{1}$ be the solution of (36) with $\epsilon>0$ and $f \in \mathcal{U}_{s+\epsilon}^{-1}$. Assume that $s+\tilde{\epsilon}+\epsilon<\frac{\lambda_{1}^{2 \theta}}{2}$ and $-s-\epsilon<\frac{\lambda_{K+1}^{2 \theta}}{2}$ for some $\tilde{\epsilon}>0$. Consider the weights $\rho$ defined in (7). We have for all $p>0$ and $\ell \leq 2$ that

$$
\left|u-u_{s}^{N, K, h}\right|_{\mathcal{U}_{s}^{1}} \leq C_{*}\left\{\max \left\{\frac{1}{1+(N+1) \lambda_{1}}, \frac{1}{1+\lambda_{K+1}}\right\}^{q}|u|_{\mathcal{U}_{p ; \rho, s+\tilde{\epsilon}+\epsilon}^{1}}+\hat{C} h^{\ell-1}\|u\|_{\mathcal{U}_{s+\tilde{\epsilon}+\epsilon}^{\ell}}\right\},
$$

where $C_{*}=C_{*}(s, \epsilon, \tilde{\epsilon})=1+e^{\frac{C_{\theta}^{2}}{\epsilon}} e^{\frac{C_{\theta}^{2}}{\tilde{\epsilon}}} \prod_{j=K+1}^{\infty} \sigma_{j}(-s-\epsilon)$ and $\hat{C}$ is is the Clement finite element interpolation constant on the space $X_{0}^{h}(\mathcal{O})$.

In this a priori error estimate (as usual in finite element analysis) some regularity is assumed. It is assumed that the norm $|u|_{\mathcal{U}_{p ; \rho, s+\tilde{\epsilon}+\epsilon}^{1}}$ is finite. In the rest of the paper we study under what conditions this is true. The proof of the a priori error estimate above can be found in [14]. The argument uses the weighted chaos norm definition of the spaces $\mid u_{\mathcal{U}_{p ; \rho, s}^{1}}$. The weights are defined in (7). Obtaining regularity results using this characterization of $\mathcal{U}_{p ; \rho, s}^{1}$ is complicated since the chaos expansion of the solution is not available. Instead, we will use the results presented in Section 3 that give a Gaussian-Sobolev norm characterization of the space $\mathcal{U}_{p ; \rho, s}^{1}$. We also mention that more general a priori error estimates are obtained in [14] that hold for any weighted chaos expansion. One important example is Given by Kondratiev type weighted chaos spaces. Unfortunately, obtaining regularity results in Kondratiev spaces is more complicated, see Section 3.4.1.

\subsection{Stochastic regularity}

We recall the definition of the tensor product space,

$$
\mathcal{U}_{p ; \rho, s}^{1}=H^{1}(\mathcal{O}) \otimes \mathcal{S}_{p ; \rho, s}
$$

For $u(x, \omega)=\sum_{\boldsymbol{\alpha} \in \mathcal{J}} u_{\boldsymbol{\alpha}, s}(x) H_{\boldsymbol{\sigma}(s)^{2}, \boldsymbol{\alpha}}(\omega),(x, \omega) \in \mathcal{O} \times \mathcal{S}^{\prime}$ we denote

$$
\|u\|_{\mathcal{U}_{p ; \rho, s}^{m}}^{2}:=\sum_{\boldsymbol{\alpha} \in \mathcal{J}} \boldsymbol{\alpha} ! \rho(\boldsymbol{\alpha}, p)^{2} \boldsymbol{\sigma}^{2 \boldsymbol{\alpha}}\left\|u_{\boldsymbol{\alpha}}\right\|_{H^{m}(\mathcal{O})}^{2},
$$

with $\rho$ defined in (7). For $k \in \mathbb{N}$ and $\boldsymbol{q} \in \mathbb{R}^{k}$ we also introduce (see Definition 5)

$$
\left\|D^{k} u\right\|_{m, \boldsymbol{q} ; s}^{2}=\sum_{\ell_{1}, \ell_{2}, \ldots, \ell_{k}} \lambda_{\ell_{1}}^{2 q_{1}} \ldots \lambda_{\ell_{k}}^{2 q_{k}}\left\|\partial_{\ell_{1}} \ldots \partial_{\ell_{k}} u\right\|_{H^{m}(\mathcal{O}) \times\left(L^{2}\right)_{s}}^{2} .
$$

From Theorem 12 and the definition of $\|\cdot\|_{\frac{k}{2} ; \rho, s}$ in (5) with $\rho$ defined in (7) we have the equality

$$
\|u\|_{\mathcal{U}_{\frac{k}{2} ; \rho, s}^{m}}^{2}=\|u\|_{\mathcal{U}_{s}^{m}}^{2}+\sum_{i=1}^{k} \sum_{R \in P^{k, i}}\left\|D^{i} u\right\|_{m, R\left(\frac{1}{2} \mathbf{1}_{k}\right) ; s^{*}}^{2}
$$

Now we study the behavior of the solution according to the regularity in the $\omega$ variable of the right-hand side data $f$. In the following result we control the 
norm of a $\omega$-partial derivative of the solution in terms of the norm of the $\omega$ partial derivatives of the forcing term. Before estating the result, we introduce needed notation. We defined the set $I(k, i)$ by

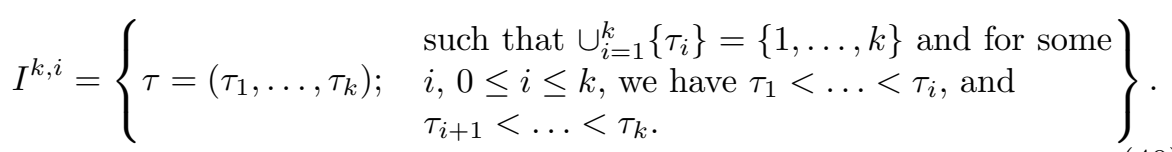

Lemma 20. Let $s \in \mathbb{R}$ and $\epsilon>0$, and let $u \in \widehat{\mathcal{U}}_{s}^{1}$ be the solution of (36) with right-hand side $f \in \mathcal{U}_{s+2 k \epsilon^{\prime}+\epsilon}^{-1}$. Let us assume that for $k \in \mathbb{N}$ and $\boldsymbol{q}=$ $\left(q_{1}, \ldots q_{k}\right) \in \mathbb{R}^{k}$ and $\epsilon^{\prime}>0$

$$
\left\|D^{i} f\right\|_{-1,\left(q_{\tau_{1}}, \ldots, q_{\tau_{i}}\right) ; s+2(k-i) \epsilon^{\prime}+\epsilon}^{2}<\infty \quad \text { for all } \quad 0 \leq i \leq k
$$

and $\phi$ satisfies

$$
\pi_{\boldsymbol{q}}(\phi):=\max _{1 \leq i \leq k} \max _{x \in \mathcal{O}}\left\|\phi_{x}\right\|_{q_{i}}<\infty
$$

Then,

$$
\left\|D^{k} \nabla u\right\|_{0, \boldsymbol{q} ; s}^{2} \leq \tilde{C}\left(\epsilon, \epsilon^{\prime}, k, \phi\right)\left(\sum_{i=0}^{k} \sum_{\tau \in I^{k, i}}\left\|D^{i} f\right\|_{-1,\left(q_{\tau_{1}}, \ldots, q_{\tau_{i}}\right) ; s+2(k-i) \epsilon^{\prime}+\epsilon}^{2}\right)
$$

where the constant $\tilde{C}\left(\epsilon, \epsilon^{\prime}, k, \phi\right)$ is given by

$$
\tilde{C}\left(\epsilon, \epsilon^{\prime}, k, \phi\right)=2^{k(k+1)} C^{2} e^{\frac{C_{\theta}^{2}}{\epsilon}} \max \left\{1, \pi_{\boldsymbol{q}}(\phi) C e^{\frac{C_{\theta}^{2}}{\epsilon^{\prime}}}\right\}^{2 k},
$$

where $C=\sqrt{1+C_{\text {poin }}}$ and $C_{\text {poin }}$ is the Poincare inequality constant which depends on $\mathcal{O}$. The set $I^{k, i}$ is defined in (40).

Proof. We first show the theorem holds for $k=1$, then we proceed by induction on the order of the derivatives $k$.

Assume that $u$ is a solution of (36). For almost sure all $\omega$ we have for all $v \in H_{0}^{1}(\mathcal{O})$

$$
\int_{\mathcal{O}} e^{\left\langle\omega, \phi_{x}\right\rangle} \nabla u(x, \omega) \nabla v(x) d x=\int_{\mathcal{O}} f v .
$$

Note that $\partial_{\ell} e^{\left\langle\omega, \phi_{x}\right\rangle}=\left\langle\phi_{x}, \eta_{\ell}\right\rangle e^{\left\langle\omega, \phi_{x}\right\rangle}$. Taking partial derivative in (43) we get

$$
\begin{aligned}
\int_{\mathcal{O}} e^{\left\langle\omega, \phi_{x}\right\rangle} \nabla \partial_{\ell} u(x, \omega) \nabla v(x) d x d \mu= & \int_{\mathcal{O}} \partial_{\ell} f(x, \omega) v(x) d x d \mu \\
& -\int_{\mathcal{O}} e^{\left\langle\omega, \phi_{x}\right\rangle}\left\langle\phi_{x}, \eta_{\ell}\right\rangle \nabla u(x, \omega) \nabla v(x) d x d \mu .
\end{aligned}
$$

Define $\Phi_{\ell}(x, \omega)=\left\langle\phi_{x}, \eta_{\ell}\right\rangle \nabla u(x, \omega)$ and by using similar arguments as in (48) below, we have $\Phi_{\ell} \in \mathcal{U}_{s+\epsilon^{\prime}+\epsilon}^{0}=\left(L_{2}\right)_{s+\epsilon^{\prime}+\epsilon}$. Integrating in $\mathcal{S}^{\prime}$ we see that $\partial_{\ell} u$ is 
the solution of the weak problem

$$
\left\{\begin{array}{l}
\text { Find } \partial_{\ell} u \in \widehat{\mathcal{U}}_{s}^{1} \text { such that } \\
a\left(\partial_{\ell} u, v\right)=G(v) \text { for all } v \in \widehat{\mathcal{U}}_{-s-\epsilon}^{1}
\end{array}\right.
$$

where the right-hand side is defined by

$$
G(v)=\int_{\mathcal{O} \times \mathcal{S}^{\prime}} \partial_{\ell} f(x, \omega) v(x, \omega) d x d \mu-\int_{\mathcal{O}} e^{\left\langle\omega, \phi_{x}\right\rangle} \Phi_{\ell}(x, \omega) \nabla v(x, \omega) d x d \mu .
$$

To bound $\left\|\partial_{\ell} u\right\|_{\mathcal{U}_{s}^{1}}$ we need first to estimate $\|G\|_{\mathcal{U}_{s+\epsilon}^{-1}}$. Note that from (33) and $2\left\langle\omega, \phi_{x}\right\rangle \leq \epsilon^{\prime}\|\omega\|_{-\theta}^{2}+C_{\theta}^{2} / \epsilon^{\prime}$ we obtain

$$
\begin{aligned}
\int_{\mathcal{O}} e^{\left\langle\omega, \phi_{x}\right\rangle} \Phi_{\ell}(x, \omega) \nabla v(x, \omega) d x d \mu \leq & \left(\int_{\mathcal{O}} e^{2\left\langle\omega, \phi_{x}\right\rangle+(s+\epsilon)\|\omega\|_{-\theta}^{2}} \Phi_{\ell}(x, \omega)^{2} d x d \mu\right)^{\frac{1}{2}} \\
& \left(\int_{\mathcal{O}} e^{(-s-\epsilon)\|\omega\|_{-\theta}^{2}} \nabla v(x, \omega)^{2} d x d \mu\right)^{\frac{1}{2}} \\
\leq & e^{\frac{C_{\theta}^{2}}{2 \epsilon^{\prime}}}\left\|\Phi_{\ell}\right\|_{s+\epsilon^{\prime}+\epsilon}\|v\|_{\mathcal{U}_{-s-\epsilon}^{1}}
\end{aligned}
$$

and then

$$
\|G\|_{\mathcal{U}_{s+\epsilon}^{-1}} \leq\left\|\partial_{\ell} f\right\|_{\mathcal{U}_{s+\epsilon}^{-1}}+e^{\frac{C_{\theta}^{2}}{2 \epsilon^{\prime}}}\left\|\Phi_{\ell}\right\|_{s+\epsilon^{\prime}+\epsilon}
$$

Using this bound and Lemma 17 applied to the weak problem (45) we have that

$$
\left\|\partial_{\ell} u\right\|_{\mathcal{U}_{s}^{1}} \leq C e^{\frac{C_{\theta}^{2}}{2 \epsilon}}\left(\left\|\partial_{\ell} f\right\|_{\mathcal{U}_{s+\epsilon}^{-1}}+e^{\frac{C_{\theta}^{2}}{2 \epsilon^{\prime}}}\left\|\Phi_{\ell}\right\|_{s+\epsilon^{\prime}+\epsilon}\right) .
$$

We can now estimate $\left\|D^{1} u\right\|_{1, q_{1} ; s}^{2}$ in Definition (5) as follows:

$$
\begin{aligned}
\left\|D^{1} u\right\|_{1, q_{1} ; s}^{2} & =\sum_{\ell=1}^{\infty} \lambda_{\ell}^{2 q_{1}}\left\|\partial_{\ell} \nabla u\right\|_{\left(L^{2}\right)_{s}}^{2} \\
& \leq 2 C^{2} e^{\frac{C_{\theta}^{2}}{\epsilon}}\left(\sum_{\ell=1}^{\infty} \lambda_{\ell}^{2 q_{1}}\left\|\partial_{\ell} f\right\|_{\mathcal{U}_{s+\epsilon}^{-1}}^{2}+e^{\frac{C_{\theta}^{2}}{\epsilon^{\prime}}} \sum_{\ell=1}^{\infty} \lambda_{\ell}^{2 q_{1}}\left\|\Phi_{\ell}\right\|_{s+\epsilon^{\prime}+\epsilon}^{2}\right) \\
& =2 C^{2} e^{\frac{C_{\theta}^{2}}{\epsilon}}\left(\left\|D^{1} f\right\|_{-1, q_{1}, s+\epsilon}^{2}+e^{\frac{C_{\theta}^{2}}{\epsilon^{\prime}}} \sum_{\ell=1}^{\infty} \lambda_{\ell}^{2 q_{1}}\left\|\Phi_{\ell}\right\|_{s+\epsilon^{\prime}+\epsilon}^{2}\right) .
\end{aligned}
$$

To estimate the last term in (47) observe that

$$
\begin{aligned}
\sum_{\ell=1}^{\infty} \lambda_{\ell}^{2 q_{1}}\left\|\Phi_{\ell}\right\|_{s+\epsilon^{\prime}+\epsilon}^{2} & =\int_{\mathcal{S}^{\prime} \times \mathcal{O}} \sum_{\ell=1}^{\infty} \lambda_{\ell}^{2 q_{1}}\left\langle\phi_{x}, \eta_{\ell}\right\rangle^{2}|\nabla u(x, \omega)|^{2} e^{\left(s+\epsilon^{\prime}+\epsilon\right)\|\omega\|_{-\theta}^{2}} d x d \mu(\omega) \\
& =\int_{\mathcal{S}^{\prime} \times \mathcal{O}}\left\|\phi_{x}\right\|_{q_{1}}^{2}|\nabla u(x, \omega)|^{2} e^{\left(s+\epsilon^{\prime}+\epsilon\right)|\omega|_{-\theta}^{2}} d x d \mu(\omega) \\
& \leq \max _{x \in \mathcal{O}}\left\|\phi_{x}\right\|_{q_{1}}^{2} \int_{\mathcal{S}^{\prime} \times \mathcal{O}}|\nabla u(x, \omega)|^{2} e^{\left(s+\epsilon^{\prime}+\epsilon\right)|\omega|_{-\theta}^{2}} d x d \mu(\omega) \\
& =\pi_{q_{1}}^{2}\|u\|_{\mathcal{U}_{s+\epsilon^{\prime}+\epsilon}^{1}}^{2}
\end{aligned}
$$


Here and below, in order to simplify notation we have written $\pi_{q_{1}}=\pi_{q_{1}}(\phi)$. By inserting Equation (48) in (47) we obtain

$$
\left\|D^{1} u\right\|_{1, q_{1} ; s}^{2} \leq 2 C^{2} e^{\frac{C_{\theta}^{2}}{\epsilon}}\left(\left\|D^{1} f\right\|_{-1, q_{1} ; s+\epsilon}^{2}+\pi_{q_{1}}^{2} e^{\frac{C_{\theta}^{2}}{\epsilon^{\prime}}}\|u\|_{\mathcal{U}_{s+\epsilon^{\prime}+\epsilon}^{1}}^{2}\right) .
$$

Using the estimate $\|u\|_{\mathcal{U}_{s+\epsilon^{\prime}+\epsilon}^{1}}^{2} \leq C^{2} e^{C_{\theta}^{2} / \epsilon^{\prime}}\|f\|_{\mathcal{U}_{s+2 \epsilon^{\prime}+\epsilon}^{-1}}^{2}$, see Lemma 17, we obtain

$$
\begin{aligned}
\left\|D^{1} u\right\|_{1, q_{1} ; s}^{2} & \leq 2 C^{2} e^{\frac{C_{\theta}^{2}}{\epsilon}}\left(\left\|D^{1} f\right\|_{-1, q_{1} ; s+\epsilon}^{2}+\pi_{q_{1}}^{2} C^{2} e^{\frac{2 C_{\theta}^{2}}{\epsilon^{\prime}}}\|f\|_{\mathcal{U}_{s+2 \epsilon^{\prime}+\epsilon}^{-1}}^{2}\right) \\
& \leq 2 C^{2} e^{\frac{C_{\theta}^{2}}{\epsilon}} \max \left\{1, \pi_{q_{1}} C e^{\frac{C_{\theta}^{2}}{\epsilon^{\prime}}}\right\}^{2}\left(\left\|D^{1} f\right\|_{-1, q_{1} ; s+\epsilon}^{2}+\|f\|_{\mathcal{U}_{s+2 \epsilon^{\prime}+\epsilon}^{-1}}\right)
\end{aligned}
$$

which finish the proof for the case $k=1$.

Now assume that the result holds valid for every $0 \leq i<k$. The main induction step argument is similar to the case $k=1$. We will:

1. Deduce a weak problem whose solution is a partial derivative of order $k$ of $u$; see (49).

2. Apply Lemma 17 to estimate the norm of each partial derivative of order $k$ of $u$, and use Definition 5 to estimate the norm of $D^{k} u$ in term of lower order derivatives of $u$; see (51).

3. Use the induction argument; see (52).

Step 1. Using the Leibniz rule we have

$$
\begin{aligned}
& \partial_{\ell_{1}} \ldots \partial_{\ell_{k}}\left(e^{\left\langle\omega, \phi_{x}\right\rangle} \nabla u(x, \omega)\right)= \\
& e^{\left\langle\omega, \phi_{x}\right\rangle} \sum_{i=0}^{k} \sum_{\tau \in I^{k, i}}\left(\nabla \partial_{\ell_{\tau_{1}}} \cdots \partial_{\ell_{\tau_{i}}} u(x, \omega)\right) a_{\ell_{\tau_{i+1}}} \cdots a_{\ell_{\tau_{k}}} \\
& =e^{\left\langle\omega, \phi_{x}\right\rangle}\left(\partial_{\ell_{1}} \ldots \partial_{\ell_{k}} \nabla u(x, \omega)+\sum_{i=0}^{k-1} \sum_{\tau \in I^{k, i}} \Phi_{\ell_{\tau_{1}} \cdots \ell_{\tau_{k}}}^{(i), \tau}(x, \omega)\right)
\end{aligned}
$$

where $a_{\ell}(x)=\left\langle\phi_{x}, \eta_{\ell}\right\rangle$ and the set $I^{k, i}$ is defined in (40). We also have defined

$$
\Phi_{\ell_{\tau_{1}} \cdots \ell_{\tau_{k}}}^{(i), \tau}(x, \omega):=\left(\nabla \partial_{\ell_{\tau_{1}}} \cdots \partial_{\ell_{\tau_{i}}} u(x, \omega)\right) a_{\ell_{\tau_{i+1}}} \cdots a_{\ell_{\tau_{k}}} .
$$

From (43) we get

$$
\begin{aligned}
& \int_{\mathcal{O}} e^{\left\langle\omega, \phi_{x}\right\rangle} \partial_{\ell_{1}} \ldots \partial_{\ell_{k}} \nabla u(x, \omega) \nabla v(x) d x d \mu=\int_{\mathcal{O}} \partial_{\ell_{1}} \ldots \partial_{\ell_{k}} f(x, \omega) v(x) d x d \mu \\
& -\int_{\mathcal{O}} e^{\left\langle\omega, \phi_{x}\right\rangle} \sum_{i=0}^{k-1} \sum_{\tau \in I^{k, i}} \Phi_{\ell_{\tau_{1}} \ldots \ell_{\tau_{k}}}^{(i), \tau}(x, \omega) \nabla v(x) d x d \mu
\end{aligned}
$$


As above we have that $\partial_{\ell_{1}} \ldots \partial_{\ell_{k}} u$ is the solution of of the weak problem

$$
\left\{\begin{array}{l}
\text { Find } \partial_{\ell_{1}} \ldots \partial_{\ell_{k}} u \in \widehat{\mathcal{U}}_{s} \text { such that } \\
a\left(\partial_{\ell_{1}} \ldots \partial_{\ell_{k}} u, v\right)=G(v) \text { for all } v \in \widehat{\mathcal{U}}_{-s-\epsilon}^{1}
\end{array}\right.
$$

with a new right-hand side

$$
\begin{aligned}
G(v)= & \int_{\mathcal{S}^{\prime} \times \mathcal{O}} \partial_{\ell_{1}} \ldots \partial_{\ell_{k}} f(x, \omega) \nabla v(x, \omega) d x d \mu- \\
& \sum_{i=0}^{k-1} \sum_{\tau \in I^{k, i}} \int_{\mathcal{S}^{\prime} \times \mathcal{O}} e^{\left\langle\omega, \phi_{x}\right\rangle} \Phi_{\ell_{\tau_{1} \ldots \ell_{\tau_{k}}}^{(i), \tau}}(x, \omega) \nabla v(x, \omega) d x d \mu .
\end{aligned}
$$

Step 2. In order to estimate $\|G\|_{s+\epsilon}$ we estimate each term in (50) above. For each $i$ and $\tau \in I^{k, i}$ we have

$\int_{\mathcal{S}^{\prime} \times \mathcal{O}} e^{\left\langle\omega, \phi_{x}\right\rangle} \Phi_{\ell_{\tau_{1} \cdots \ell_{\tau_{k}}}^{(i), \tau}}(x, \omega) \nabla v(x, \omega) d x d \mu \leq e^{\frac{C_{\theta}^{2}}{2 \epsilon^{\prime}}}\left\|\Phi_{\ell_{\tau_{1}} \cdots \ell_{\tau_{k}}}^{(i), \tau}\right\|_{s+\epsilon^{\prime}+\epsilon}\|v\|_{\mathcal{U}_{-s-\epsilon}^{1}}$.

Then from Lemma 17 applied to problem (49) with the right-hand side $G$ in (50) we get

$\left\|\partial_{\ell_{1}} \ldots \partial_{\ell_{k}} u\right\|_{\mathcal{U}_{s}^{1}} \leq C e^{\frac{C_{\theta}^{2}}{2 \epsilon}}\left(\left\|\partial_{\ell_{1}} \ldots \partial_{\ell_{k}} f\right\|_{\mathcal{U}_{s+\epsilon}^{-1}}+e^{\frac{C_{\theta}^{2}}{2 \epsilon^{\prime}}} \sum_{i=0}^{k-1} \sum_{\tau \in I^{k, i}}\left\|\Phi_{\ell_{\tau_{1}} \ldots \ell_{\tau_{k}}}^{(i), \tau}\right\|_{s+\epsilon^{\prime}+\epsilon}\right)$

and using $\sum_{i=0}^{k-1} \sum_{\tau \in I^{k, i}} 1=\sum_{i=0}^{k-1}\left(\begin{array}{c}k \\ i\end{array}\right)=2^{k}-1$, we obtain

$$
\begin{aligned}
\left\|D^{k} \nabla u\right\|_{0, \boldsymbol{q} ; s}^{2}= & \sum_{\ell_{1}, \cdots, \ell_{k}} \lambda_{\ell_{1}}^{2 q_{1}} \cdots \lambda_{\ell_{k}}^{2 q_{k}}\left\|\partial_{\ell_{1}} \ldots \partial_{\ell_{k}} u\right\|_{\mathcal{U}_{s}^{1}}^{2} \\
\leq & 2^{k} C^{2} e^{\frac{C_{\theta}^{2}}{\epsilon}}\left(\sum_{\ell_{1}, \cdots, \ell_{k}} \lambda_{\ell_{1}}^{2 q_{1}} \cdots \lambda_{\ell_{k}}^{2 q_{k}}\left\|\partial_{\ell_{1}} \ldots \partial_{\ell_{k}} f\right\|_{\mathcal{U}_{s+\epsilon}^{-1}}^{2}+\right. \\
& \left.e^{\frac{C_{\theta}^{2}}{\epsilon^{\prime}}} \sum_{\ell_{1}, \cdots, \ell_{k}} \lambda_{\ell_{1}}^{2 q_{1}} \ldots \lambda_{\ell_{k}}^{2 q_{k}} \sum_{i=0}^{k-1} \sum_{\tau \in I^{k, i}}\left\|\Phi_{\ell_{\tau_{1}} \ldots \ell_{\tau_{k}}}^{(i), \tau}\right\|_{s+\epsilon^{\prime}+\epsilon}^{2}\right) \\
\leq & 2^{k} C^{2} e^{\frac{C_{\theta}^{2}}{\epsilon}}\left(\left\|D^{k} f\right\|_{-1, \boldsymbol{q} ; s+\epsilon}^{2}+\right. \\
& \left.e^{\frac{C_{\theta}^{2}}{\epsilon^{\prime}}} \sum_{i=0}^{k-1} \sum_{\tau \in I^{k, i}} \sum_{\ell_{1}, \cdots, \ell_{k}} \lambda_{\ell_{1}}^{2 q_{1}} \cdots \lambda_{\ell_{k}}^{2 q_{k}}\left\|\Phi_{\ell_{\tau_{1}} \ldots \ell_{\tau_{k}}}^{(i), \tau}\right\|_{s+\epsilon^{\prime}+\epsilon}^{2}\right) .
\end{aligned}
$$

Finally note that

$$
\begin{aligned}
& \lambda_{\ell_{1}}^{2 q_{1}} \cdots \lambda_{\ell_{k}}^{2 q_{k}}\left\|\Phi_{\ell_{\tau_{1}} \cdots \ell_{\tau_{k}}}^{(i), \tau}\right\|_{\left(s+\epsilon^{\prime}+\epsilon\right)}^{2}=\lambda_{\ell_{\tau_{1}}}^{2 q_{\tau_{1}}} \cdots \lambda_{\ell_{\tau_{k}}}^{2 q_{\tau_{k}}}\left\|\Phi_{\ell_{\tau_{1}} \cdots \ell_{\tau_{k}}}^{(i), \tau}\right\|_{\left(s+\epsilon^{\prime}+\epsilon\right)}^{2} \\
& =\int_{\mathcal{S}^{\prime} \times \mathcal{O}}\left(\lambda_{\ell_{\tau_{1}}}^{2 q_{\tau_{1}}} \cdots \lambda_{\ell_{\tau_{i}}}^{2 q_{\tau_{\tau_{i}}}}\left|\partial_{\ell_{\tau_{1}}} \cdots \partial_{\ell_{\tau_{i}}} \nabla u(x, \omega)\right|^{2}\right) \cdot\left(\lambda_{\ell_{\tau_{i+1}}}^{2 q_{\tau_{i+1}}} \cdots\right. \\
& \left.\quad \lambda_{\ell_{\tau_{i+1}} q_{\tau_{i+1}}}^{2} a_{\ell_{\tau_{i+1}}}^{2}(x) \cdots a_{\ell_{\tau_{k}}}^{2}(x)\right) e^{\left(s+\epsilon^{\prime}+\epsilon\right)\|\omega\|_{-\theta}^{2}} d \mu d x
\end{aligned}
$$


implies

$$
\begin{aligned}
& \sum_{\ell_{1}, \cdots, \ell_{k}} \lambda_{\ell_{1}}^{2 q_{1}} \cdots \lambda_{\ell_{k}}^{2 q_{k}}\left\|\Phi_{\ell_{\tau_{1}} \cdots \ell_{\tau_{k}}}^{(i), \tau_{s}}\right\|_{s+\epsilon^{\prime}+\epsilon}^{2} \\
& =\max _{x \in \mathcal{O}}\left\|\phi_{x}\right\|_{q_{\tau_{i+1}}}^{2} \cdots\left\|\phi_{x}\right\|_{q_{\tau_{k}}}^{2}\left\|D^{i} \nabla u\right\|_{0,\left(q_{\tau_{1}}, \ldots, q_{\tau_{i}}\right), s+\epsilon^{\prime}+\epsilon}^{2} \\
& \leq \pi_{\boldsymbol{q}}^{2(k-i)}\left\|D^{i} \nabla u\right\|_{0,\left(q_{\tau_{1}}, \ldots, q_{\tau_{i}}\right) ; s+\epsilon^{\prime}+\epsilon}^{2} .
\end{aligned}
$$

Here and below, in order to simplify the notation we have written $\pi_{\boldsymbol{q}}=\pi_{\boldsymbol{q}}(\phi)$. Summarizing

$$
\begin{aligned}
\left\|D^{k} \nabla u\right\|_{\boldsymbol{q} ; s}^{2} \leq & 2^{k} C^{2} e^{\frac{C_{\theta}^{2}}{\epsilon}}\left(\left\|D^{k} f\right\|_{-1, \boldsymbol{q} ; s+\epsilon}^{2}+\right. \\
& \left.e^{\frac{C_{\theta}^{2}}{\epsilon^{\prime}}} \sum_{i=0}^{k-1} \pi_{\boldsymbol{q}}^{2(k-i)} \sum_{\tau \in I^{k, i}}\left\|D^{i} \nabla u\right\|_{0,\left(q_{\tau_{1}}, \ldots, q_{\tau_{i}}\right) ; s+\epsilon^{\prime}+\epsilon}^{2}\right) .
\end{aligned}
$$

Step 3. We have from the induction argument, i.e., (41) holds with $k$ replaced $i, s$ replaced by $s+\epsilon^{\prime}+\epsilon$ and $\epsilon$ replaced by $\epsilon^{\prime}$,

$$
\begin{aligned}
& e^{\frac{C_{\theta}^{2}}{\epsilon^{\prime}}} \sum_{i=0}^{k-1} \pi_{\boldsymbol{q}}^{2(k-i)} \sum_{\tau \in I^{k, i}}\left\|D^{i} \nabla u\right\|_{0,\left(q_{\tau_{1}}, \ldots, q_{\tau_{i}}\right) ; s+\epsilon^{\prime}+\epsilon}^{2} \\
\leq & e^{\frac{C_{\theta}^{2}}{\epsilon^{\prime}}} \sum_{i=0}^{k-1} \pi_{\boldsymbol{q}}^{2(k-i)} 2^{i(i+1)} C^{2} e^{\frac{C_{\theta}^{2}}{\epsilon^{\prime}}} \max \left\{1, \pi_{\boldsymbol{q}} C e^{\frac{C_{\theta}^{2}}{\epsilon^{\prime}}}\right\}^{2 i}( \\
& \left.\sum_{\tau \in I^{k, i}} \sum_{j=0}^{i} \sum_{\tau^{\prime} \in I^{i, j}}\left\|D^{j} f\right\|_{-1,\left(q_{\tau_{1}^{\prime}}, \ldots, q_{\tau_{\tau_{j}^{\prime}}}\right) ;\left(s+\epsilon^{\prime}+\epsilon\right)+\left(2(i-j) \epsilon^{\prime}+\epsilon^{\prime}\right)}^{2}\right)
\end{aligned}
$$

Now we use the fact that the total number of terms in the sum $\sum_{i=0}^{k-1} \sum_{\tau \in I^{k, i}}$ is $2^{k}-1$ to get

$$
\begin{aligned}
& \sum_{i=0}^{k-1} \sum_{\tau \in I^{k, i}} \sum_{j=0}^{i} \sum_{\tau^{\prime} \in I^{i, j}}\left\|D^{j} f\right\|_{-1,\left(q_{\tau_{\tau_{1}^{\prime}}}, \ldots, q_{\tau_{\tau_{j}^{\prime}}}\right), s+2(i-j) \epsilon^{\prime}+\epsilon}^{2} \leq \\
& \sum_{i=0}^{k-1} \sum_{\tau \in I^{k, i}} \sum_{j=0}^{i} \sum_{\tau^{\prime} \in I^{i, j}}\left\|D^{j} f\right\|_{-1,\left(q_{\tau_{\tau_{1}^{\prime}}}, \ldots, q_{\tau_{\tau_{j}^{\prime}}}\right), s+2(k-j) \epsilon^{\prime}+\epsilon}^{2} \leq \\
& \sum_{i=0}^{k-1}\left(2^{k}-1\right) \sum_{\tau \in I^{k, j}}\left\|D^{j} f\right\|_{-1,\left(q_{\tau_{1}}, \ldots, q_{\tau_{j}}\right), s+2(k-j) \epsilon^{\prime}+\epsilon}^{2} .
\end{aligned}
$$

Using that $C e^{\frac{C_{\theta}^{2}}{\epsilon^{\prime}}}>1$, hence $C e^{\frac{C_{\theta}^{2}}{\epsilon^{\prime}}} \leq\left(C e^{\frac{C_{\theta}^{2}}{\epsilon^{\prime}}}\right)^{2(k-i)}$ for $0 \leq i \leq k-1$, and together with (52) yields

$$
e^{\frac{C_{\theta}^{2}}{\epsilon^{\prime}}} \sum_{i=0}^{k-1} \pi_{\boldsymbol{q}}^{2(k-i)} \sum_{\tau \in I^{k, i}}\left\|D^{i} \nabla u\right\|_{0,\left(q_{\tau_{1}}, \ldots, q_{\tau_{i}}\right) ; s+\epsilon^{\prime}+\epsilon}^{2}
$$




$$
\begin{aligned}
\leq & \left(2^{k}-1\right) \max \left\{1, \pi_{\boldsymbol{q}} C e^{\frac{C_{\theta}^{2}}{\epsilon^{\prime}}}\right\}^{2 k}( \\
& \left.\sum_{i=0}^{k-1} 2^{i(i+1)} \sum_{\tau \in I^{k, i}}\left\|D^{i} f\right\|_{-1,\left(q_{\tau_{1}}, \ldots, q_{\tau_{i}}\right), s+2(k-i) \epsilon^{\prime}+\epsilon}^{2}\right) \\
\leq & 2^{(k-1) k}\left(2^{k}-1\right) \max \left\{1, \pi_{\boldsymbol{q}} C e^{\frac{C_{\theta}^{2}}{\epsilon^{\prime}}}\right\}^{2 k}( \\
& \left.\sum_{\tau \in I^{k, \ell}}\left\|D^{i} f\right\|_{-1,\left(q_{\tau_{1}}, \ldots, q_{\tau_{i}}\right), s+2(k-i) \epsilon^{\prime}+\epsilon}^{2}\right) .
\end{aligned}
$$

Inserting (54) in (51) we get

$$
\begin{gathered}
\left\|D^{k} \nabla u\right\|_{\boldsymbol{q} ; s}^{2} \leq 2^{k}\left(1+2^{(k-1) k}\left(2^{k}-1\right)\right) C^{2} e^{\frac{C_{\theta}^{2}}{\epsilon}} \max \left\{1, \pi_{\boldsymbol{q}} C e^{\frac{C_{\theta}^{2}}{\epsilon^{\theta}}}\right\}^{2 k}( \\
\left.\sum_{i=0}^{k} \sum_{\tau \in I^{k, i}}\left\|D^{i} f\right\|_{-1,\left(q_{\tau_{1}}, \ldots, q_{\tau_{i}}\right) ; s+2(k-i) \epsilon^{\prime}+\epsilon}^{2}\right)
\end{gathered}
$$

and (41) follows by using $2^{k}\left(1+2^{(k-1) k}\left(2^{k}-1\right)\right) \leq 2^{k(k+1)}$.

Corollary 21. Let $s \in \mathbb{R}$ and $\epsilon>0$, and let $u \in \widehat{\mathcal{U}}_{s}^{1}$ be the solution of (36) with right-hand side $f \in \mathcal{U}_{s+2 k \epsilon^{\prime}+\epsilon}^{-1}$. Let us assume that for $k \in \mathbb{N}$ and $\boldsymbol{q}=$ $\left(q_{1}, \ldots q_{k}\right) \in \mathbb{R}^{k}$ we have $\left\|\phi_{x}\right\|_{q_{i}}<\infty$ for $1 \leq i \leq k$ and $\|f\|_{\mathcal{U}_{|\boldsymbol{q}| ;, t}^{-1}}<\infty$ where $|\boldsymbol{q}|=\sum_{i=1}^{k} q_{i}, \rho$ is defined by (7), $\epsilon^{\prime}>0$ and $t=s+2 k \epsilon^{\prime}+\epsilon$. Then

$$
\left\|D^{k} \nabla u\right\|_{0, \boldsymbol{q} ; s}^{2}<\tilde{C}\left(\epsilon, \epsilon^{\prime}, k, \phi\right)\|f\|_{\mathcal{U}_{|\boldsymbol{q}| ; \rho, t}^{-1}}^{2}
$$

where the constant $\tilde{C}$ is defined in (42).

Proof. From Lemma 20 and Lemma 17 we have

$$
\begin{aligned}
\left\|D^{k} \nabla u\right\|_{0, \boldsymbol{q} ; s}^{2} & \leq \tilde{C}\left(\epsilon, \epsilon^{\prime}, k, \phi\right)\left(\sum_{i=0}^{k} \sum_{\tau \in I^{k, \ell}}\left\|D^{i} f\right\|_{-1,\left(q_{\tau_{1}}, \ldots, q_{\tau_{i}}\right) ; t}^{2}\right) \\
& <\tilde{C}\left(\epsilon, \epsilon^{\prime}, k, \phi\right)\left(\sum_{i=0}^{k} \sum_{R \in P^{k, i}}\left\|D^{i} f\right\|_{-1, R(\boldsymbol{q}) ; t}^{2}\right) \\
& \leq \tilde{C}\left(\epsilon, \epsilon^{\prime}, k, \phi\right)\|f\|_{\mathcal{U}_{|\boldsymbol{q}| ;, t}^{-1}}^{1}
\end{aligned}
$$

The following result summarizes our stochastic regularity result.

Theorem 22. Let $s \in \mathbb{R}$ and $\epsilon>0$, and let $u \in \widehat{\mathcal{U}}_{s}^{1}$ be the solution of (36) with right-hand side $f \in \mathcal{U}_{s+\epsilon}^{-1}$. Let us assume that for $k \in \mathbb{N}$ we have $\left\|\phi_{x}\right\|_{\frac{k}{2}}<\infty$ where $\rho$ is defined by (7), $\epsilon^{\prime}>0$ and $t=s+2 k \epsilon^{\prime}+\epsilon$. Then $u \in \widehat{\mathcal{U}}_{\frac{k}{2} ; \rho, s}^{1}$ and

$$
|u|_{\mathcal{U}_{\frac{k}{2} ; \rho, s}^{1}} \leq \tilde{C}\left(\epsilon, \epsilon^{\prime}, k, \phi\right) B(k)\|f\|_{\mathcal{U}_{\frac{k}{2} ; \rho, t}^{-1}}^{2}
$$


where (the Bell number) $B(k)$ is the total number of partitions of the set $\{1,2, \ldots, k\}$.

Proof. According to Corollary 21 we see that

$$
\begin{aligned}
\|\nabla u\|_{\mathcal{U}_{\frac{k}{2} ; \rho, s}^{0}}^{2} & =\sum_{i=1}^{k} \sum_{R \in P^{k, i}}\left\|D^{i} \nabla u\right\|_{0, R\left(\frac{1}{2} \mathbf{1}_{k}\right) ; \rho, s}^{2} \\
& <\sum_{i=1}^{p} \sum_{R \in P^{k, i}} \tilde{C}\left(\epsilon^{\prime}, i, \phi, \mathcal{O}\right)\|f\|_{\mathcal{U}_{\left|R\left(\frac{1}{2} \mathbf{1}_{k}\right)\right| ; \rho, t}^{-1}}^{2} \\
& \leq \tilde{C}\left(\epsilon^{\prime}, k, \phi, \mathcal{O}\right) B(k)\|f\|_{\mathcal{U}_{\frac{k}{2} ; \rho, t}^{-1}}^{2},
\end{aligned}
$$

where we have used that $\left|R\left(\frac{1}{2} \mathbf{1}_{k}\right)\right|=\frac{k}{2}$ for all $R$ partition of the set $\{1,2, \ldots, k\}$.

Remark 23. Bounds for the Bell numbers $B(k)$ are know. It is know that $B(k)=\frac{1}{e} \sum_{i=1}^{\infty} \frac{i^{k}}{i !}<\left(\frac{0.792 k}{\ln (k+1)}\right)^{k}$. See [4] and references therein.

Remark 24. In the special case of $f$ being a polynomial in $\omega$, i.e., a finite sum of Fourier-Hermite polynomials with coefficients in $H^{-1}(\mathcal{O})$ we can easily verify that $f \in \mathcal{U}_{p ; \rho, s}^{-1}$ for all $p$ and all $s<\frac{\lambda_{1}^{2 \theta}}{2}$.

Next we present a result that can be directly applied to bound the first term in the a priori error estimate in Lemma 19.

Corollary 25. Let the conditions of Theorem 22 hold with $k=2 p$ and $\epsilon^{\prime}=\frac{\tilde{\epsilon}}{2 p}$. Then

$$
|u|_{\mathcal{U}_{p ; \rho, s+\tilde{\epsilon}+\epsilon}^{1}} \leq \tilde{C}\left(\epsilon, \frac{\tilde{\epsilon}}{2 p}, 2 p, \phi\right) B(2 p)\|f\|_{\mathcal{U}_{p ; \rho, s+\tilde{\epsilon}+\epsilon}^{-1}}^{2},
$$

where the constant $\tilde{C}$ is defined in (42).

\subsection{Spatial regularity}

In this section we will study the spatial regularity of the solution of (36).

Fix $\omega$ and take partial derivatives with respect to spatial coordinates $x_{i}$. In particular $\frac{\partial\left\langle\omega, \phi_{x}\right\rangle}{\partial x_{i}}=\left\langle\omega,\left(\frac{\partial \phi_{x}}{\partial x_{i}}\right)\right\rangle$, hence,

$$
\left|\frac{\partial\left\langle\omega, \phi_{x}\right\rangle}{\partial x_{i}}\right| \leq \max _{x \in \mathcal{O}}\left\|\frac{\partial \phi_{x}}{\partial x_{i}}\right\|_{\theta}\|\omega\|_{-\theta}=\tilde{\tilde{C}}_{\theta}(\phi)\|\omega\|_{-\theta}
$$

where we have denoted

$$
\tilde{\tilde{C}}_{\theta}(\phi)=\max _{1 \leq i \leq d} \max _{x \in \mathcal{O}}\left\|\frac{\partial \phi_{x}}{\partial x_{i}}\right\|_{\theta} .
$$

Since $\frac{\partial \kappa(x, \omega)}{\partial x_{i}}=\left\langle\omega,\left(\frac{\partial \phi_{x}}{\partial x_{i}}\right)\right\rangle e^{\left\langle\omega, \phi_{x}\right\rangle}$, we obtain

$$
\left|\frac{\partial \kappa}{\partial x_{i}}(x, \omega)\right| \leq \tilde{\tilde{C}}_{\theta}(\phi)\|\omega\|_{-\theta} e^{\frac{C_{\theta}^{2}}{2 \epsilon}} e^{\frac{\epsilon}{2}\|\omega\|_{-\theta}^{2}} .
$$


Remark 26. In general we have

$$
\left\|\frac{\partial \phi_{x}}{\partial x_{i}}\right\|_{\theta}^{2}=\sum_{j=1}^{\infty} \lambda_{j}^{2 \theta}\left(\frac{\partial \phi_{x}}{\partial x_{i}}, \eta_{j}\right)_{H}^{2}
$$

For the particular case of Example 16 we have $\phi_{x}(\cdot)=\phi(\cdot-x)$, hence, if we consider for simplicity the uni-dimensional case $H=L^{2}(\mathbb{R})$, we have

$$
\begin{gathered}
\left(\frac{\partial \phi_{x}}{\partial x}, \eta_{j}\right)_{H}=\int_{\mathbb{R}} \frac{\partial \phi}{\partial x}(\hat{x}-x) \eta_{j}(\hat{x}) d \hat{x}=-\int_{\mathbb{R}} \frac{\partial \phi}{\partial \hat{x}}(\hat{x}-x) \eta_{j}(\hat{x}) d \hat{x}= \\
\int_{\mathbb{R}} \phi(\hat{x}-x) \frac{\partial}{\partial \hat{x}} \eta_{j}(\hat{x}) d \hat{x}=\sqrt{\frac{j}{2}}\left(\frac{\partial \phi_{x}}{\partial x}, \eta_{j-1}\right)_{H}-\sqrt{\frac{j+1}{2}}\left(\frac{\partial \phi_{x}}{\partial x}, \eta_{j+1}\right)_{H},
\end{gathered}
$$

where we have used a recursive relation of derivative of Hermite functions. Using that $\lambda_{j}=2 j$, we obtain $\tilde{\tilde{C}}_{\theta}(\phi) \leq \check{C} C_{\theta+\frac{1}{2}}$. For the case of Example 15, we have neither the recursive relation nor $\eta_{j}$ vanishes on $\partial \mathcal{O}$; see [13] for issues on the regularity of the $\eta_{j}$ and the decaying of the $1 / \lambda_{j}$.

The following result is a particular case of Theorem 9.1, page 184 of Ladyzhenskaya and Ural'tseva [21].

Lemma 27. Consider the following elliptic problem

$$
\left\{\begin{aligned}
-\nabla \cdot(\mu(x) \nabla u(x)) & =f(x), \text { for } x \in \mathcal{O} \\
u(x) & =0, \text { on } \partial \mathcal{O} .
\end{aligned}\right.
$$

Suppose that:

1. There is constants such that

$$
0<\mu_{\min } \leq \mu(x) \leq \mu_{\max } \quad \text { for all } x \in \mathcal{O} \text { and that } \partial \mathcal{O}
$$

and

$$
\left\|\frac{\partial \mu}{\partial x_{j}}\right\|_{L_{q}(\mathcal{O})} \leq \mu_{\max } \text { with } q>d
$$

2. $\partial \mathcal{O}$ is piecewise smooth with curvature bounded below by a number K (See [21] page 174 and 175).

3. The domain $\mathcal{O}$ is of class $W_{q}^{2}$ or that $\mathcal{O}$ can be topologically mapped into a parallelepiped by a function in $W_{q}^{2}\left(\mathbb{R}^{d}\right)$ with nonzero Jacobian.

Then the problem has unique solution in $H^{2}(\mathcal{O}) \cap H_{0}^{1}(\mathcal{O})$ if $f \in L^{2}(\mathcal{O})$.

Corollary 28. Under the assumption of Lemma 27 for the Domain $\mathcal{O}$ we have that for almost all $\omega \in \Omega$ the weak solution $u(\cdot, \omega)$ of Problem (30) is an element of $H^{2}(\mathcal{O})$ if $f(\cdot, \omega) \in L^{2}(\mathcal{O})$ 
Now we only need to bound the $H^{2}$ norm of $u(\cdot, \omega)$ in terms of $\omega$. We next apply the second fundamental inequality, Lemma 8.1 in page 175 of [21] applied to the class of coefficients we consider in this paper. In order to simplify the presentation we assume that $\mathcal{O}$ is a nondegenerate $d-1$ dimension polyhedron.

Lemma 29. Assume that $\mathcal{O}$ is a nondegenerate $d-1$ dimensional polyhedron. For every function $v \in H^{2} \cap H_{0}^{1}$ we have that for every $\hat{\epsilon}>0$ and almost all $\omega \in \mathcal{S}^{\prime}$

$$
|v|_{H^{2}(\mathcal{O})}^{2} \leq 2 e^{\frac{C_{\theta}^{2}}{\epsilon}} e^{\hat{\epsilon}\|\omega\|_{-\theta}^{2}} \int_{\mathcal{O}}(\nabla \cdot \kappa \nabla v)^{2}+\frac{8 d}{\hat{\epsilon}} \tilde{\tilde{C}}_{\theta}(\phi) e^{\frac{4 C_{\theta}^{2}}{\epsilon}} e^{5 \hat{\epsilon}\|\omega\|_{-\theta}^{2}} \int_{\mathcal{O}}|\nabla v|^{2}
$$

where $C_{\theta}$ is defined in (32) and $\tilde{\tilde{C}}_{\theta}$ in (57).

Proof. Note that it is enough prove the result for smooth functions $v$. Assume $v \in C^{2}(\overline{\mathcal{O}})$ and $v=0$ on $\partial \mathcal{O}$. We have

$$
(\nabla \cdot \kappa \nabla v)^{2}=(\nabla \kappa \cdot \nabla v)^{2}+2 \kappa(\nabla \kappa \cdot \nabla v)(\Delta v)+\kappa^{2}(\Delta v)^{2} .
$$

Using two integration by parts and $v=0$ on $\partial \mathcal{O}$ we have

$$
\int_{\mathcal{O}}(\Delta v)^{2}=|v|_{H^{2}(\mathcal{O})}^{2}+\int_{\partial \mathcal{O}} \Delta v \nabla v \cdot \boldsymbol{\eta}-\sum_{i=1}^{d} \partial_{i} v \nabla\left(\partial_{i} v\right) \cdot \boldsymbol{\eta}=|v|_{H^{2}(\mathcal{O})}^{2} .
$$

To see the boundary integral vanish it is enough to compute this integral in each face of $\mathcal{O}$. Let $F$ be a face of $\mathcal{O}$. We can assume $F \subset R^{d-1} \times\{0\}$. Then $\boldsymbol{\eta}=(0, \ldots, 1) \in \mathbb{R}^{d}$. Then

$$
\int_{F} \Delta v \nabla v \cdot \boldsymbol{\eta}-\sum_{i=1}^{d} \partial_{i} v \nabla\left(\partial_{i} v\right) \cdot \boldsymbol{\eta}=\int_{F} \Delta v \partial_{d} v-\sum_{i=1}^{d} \partial_{i} v \partial_{d i}^{2} v
$$

Since $v=0$ on $F$ we have that $\partial_{i} v=0$ and $\partial_{i j} v=0$, on $F, i=1, \ldots, d-1$. Then

$$
\int_{F} \Delta v \nabla v \cdot \boldsymbol{\eta}-\sum_{i=1}^{d} \partial_{i} v \nabla\left(\partial_{i} v\right) \cdot \boldsymbol{\eta}=\int_{F} \partial_{d d} v \partial_{d} v-\partial_{d} v \partial_{d d}^{2} v=0 .
$$

Now, observe that

$$
\int_{\mathcal{O}}(\nabla \kappa \cdot \nabla v)^{2} \leq d \max _{1 \leq i \leq d}\left\|\partial_{i} \kappa\right\|_{\infty}^{2} \int_{\mathcal{O}}|\nabla v|^{2}
$$

and with $\delta=\frac{\kappa_{\min }^{2}}{2 \kappa_{\max }}>0$ we have

$$
\begin{aligned}
2 \int_{\mathcal{O}} \kappa(\nabla \kappa \cdot \nabla v)(\Delta v) & \leq \kappa_{\max }\left(\delta \int_{\mathcal{O}}(\Delta v)^{2}+\frac{1}{\delta} \int_{\mathcal{O}}(\nabla \kappa \cdot \nabla v)^{2}\right) \\
& \leq \delta \kappa_{\max }|v|_{H^{2}(\mathcal{O})}^{2}+d \max _{1 \leq i \leq d}\left\|\partial_{i} \kappa\right\|_{\infty}^{2} \frac{\kappa_{\max }}{\delta} \int_{\mathcal{O}}|\nabla v|^{2} \\
& \left.\leq \frac{\kappa_{\min }^{2}}{2}|v|_{H^{2}(\mathcal{O})}^{2}+2 d \max _{1 \leq i \leq d}\left\|\partial_{i} \kappa\right\|_{\infty}^{2} \frac{\kappa_{\max }^{2}}{\kappa_{\min }^{2}} \int_{\mathcal{O}}|\nabla v|^{2} 66\right)
\end{aligned}
$$


By combining (62), (65) and (66) we get

$$
\begin{aligned}
|v|_{H^{2}(\mathcal{O})}^{2} \leq & \frac{1}{\kappa_{\min }^{2}} \int_{\mathcal{O}}(\nabla \cdot \kappa \nabla v)^{2}+ \\
& +\frac{1}{2}|v|_{H^{2}(\mathcal{O})}^{2}+2 d \max _{1 \leq i \leq d}\left\|\partial_{i} \kappa\right\|_{\infty}^{2}\left(\frac{1}{\kappa_{\min }^{2}}+\frac{\kappa_{\max }^{2}}{\kappa_{\min }^{4}}\right) \int_{\mathcal{O}}|\nabla v|^{2}
\end{aligned}
$$

and then

$$
|v|_{H^{2}(\mathcal{O})}^{2} \leq \frac{2}{\kappa_{\min }^{2}} \int_{\mathcal{O}}(\nabla \cdot \kappa \nabla v)^{2}+4 d \max _{1 \leq i \leq d}\left\|\partial_{i} \kappa\right\|_{\infty}^{2}\left(\frac{1}{\kappa_{\min }}+\frac{\kappa_{\max }^{2}}{\kappa_{\min }^{2}}\right) \int_{\mathcal{O}}|\nabla v|^{2} .
$$

Finally, using (33), we see that

$$
\begin{aligned}
|v|_{H^{2}(\mathcal{O}) \leq}^{2} \leq & 2 e^{\frac{C_{\theta}^{2}}{\hat{\epsilon}}} e^{\hat{\epsilon}\|\omega\|_{-\theta}^{2}} \int_{\mathcal{O}}(\nabla \cdot \kappa \nabla v)^{2}+ \\
& 4 d \max _{1 \leq i \leq d}\left\|\partial_{i} \kappa\right\|_{\infty}^{2}\left(1+e^{\frac{3 C_{\theta}^{2}}{\hat{\epsilon}}} e^{3 \hat{\epsilon}\|\omega\|_{-\theta}^{2}}\right) \int_{\mathcal{O}}|\nabla v|^{2}
\end{aligned}
$$

and using (58) we get

$$
\begin{aligned}
|v|_{H^{2}(\mathcal{O}) \leq}^{2} \leq & 2 e^{\frac{C_{\theta}^{2}}{\hat{\epsilon}}} e^{\hat{\epsilon}\|\omega\|_{-\theta}^{2}} \int_{\mathcal{O}}(\nabla \cdot \kappa \nabla v)^{2}+ \\
& 4 d \tilde{\tilde{C}}_{\theta}(\phi)\|\omega\|_{-\theta}^{2} e^{\frac{C_{\theta}^{2}}{\hat{\epsilon}}} e^{\hat{\epsilon}\|\omega\|_{-\theta}^{2}}\left(1+e^{\frac{3 C_{\theta}^{2}}{\hat{\epsilon}}} e^{3 \hat{\epsilon}\|\omega\|_{-\theta}^{2}}\right) \int_{\mathcal{O}}|\nabla v|^{2}
\end{aligned}
$$

and using that $\hat{\epsilon}\|\omega\|_{-\theta}^{2}<e^{\hat{\epsilon} \mid \omega \|_{-\theta}^{2}}$, (60) follows.

We establish a bound for the second term in the a priori error estimate 19.

Theorem 30. Let $s \in \mathbb{R}$ and $\epsilon>0$, and let $u \in \widehat{\mathcal{U}}_{s}^{1}$ be the solution of (36) with right-hand side $f \in \mathcal{U}_{s+\epsilon}^{-1}$. Assume that $\mathcal{O}$ is a nondegenerate $d-1$ dimensional polyhedron, $f \in \mathcal{U}_{s+\tilde{\epsilon}+\epsilon+\hat{\epsilon}}^{0}$ and $f \in \mathcal{U}_{s+\tilde{\epsilon}+2 \epsilon+5 \hat{\epsilon}}^{-1}$ for $\tilde{\epsilon}, \epsilon, \hat{\epsilon}$ positive. Then, $u \in$ $\mathcal{U}_{s+\epsilon \tilde{+} \hat{\epsilon}}^{2}$ and

$$
\begin{aligned}
|u|_{\mathcal{U}_{s+\tilde{\epsilon}+\epsilon+\hat{\epsilon}}^{2} \leq}^{2} \leq & 2 e^{\frac{C_{\theta}^{2}}{\hat{\epsilon}}} e^{\hat{\epsilon}\|\omega\|_{-\theta}^{2}}\|f\|_{\mathcal{U}_{s+\tilde{\epsilon}+\epsilon+\hat{\epsilon}}^{0}}^{2}+ \\
& \frac{8 d}{\hat{\epsilon}} C^{2} \tilde{\widetilde{C}}_{\theta}(\phi) e^{C_{\theta}^{2}\left(\frac{4}{\hat{\epsilon}}+\frac{1}{\epsilon}\right)} e^{(4 \hat{\epsilon}+\epsilon)\|\omega\|_{-\theta}^{2}\|f\|_{\mathcal{U}_{s+\tilde{\epsilon}+2 \epsilon+5 \hat{\epsilon}}^{-1}}^{2}}
\end{aligned}
$$

where $C=\sqrt{1+C_{\text {poin }}}$ and $C_{\text {poin }}$ is the Poincaré inequality constant which depends on $\mathcal{O}, C_{\theta}$ is defined in (32) and $\tilde{\tilde{C}}_{\theta}$ in (57).

Proof. Corollary 28 and $f \in \mathcal{U}_{s+\tilde{\epsilon}+\epsilon+\hat{\epsilon}}^{0}$ imply that for almost all $\omega \in \mathcal{S}^{\prime}, u(\cdot, \omega) \in$ $H^{2}(\mathcal{O})$. The bound (67) follows by first replacing $v$ by $u$ in $(60)$, then multiply (60) by $e^{(s+\tilde{\epsilon}+\epsilon)\|\omega\|_{-\theta}^{2}}$ and integrate in $\mathcal{S}^{\prime}$, then use $\nabla \cdot \kappa \nabla u=f$ to obtain the first term of the right-hand side of (67), and use Lemma 17 to obtain the second term. 


\section{Final remarks}

We presented regularity results for stochastic elliptic equations with lognormal coefficient $\kappa$. We obtained joint spatial and stochastic regularity of solutions of the ordinary product pressure equation assuming similar regularity for the right-hand side $f(x, \omega)$ and stochastic process $\log (\kappa(x, \omega))$. Standard assumptions on the spatial domain $\mathcal{O}$ are also used. The main results in Theorem 22 and Theorem 30 which state that the solution of the pressure equation with regular data has classical $H^{1+r}$ regularity in the spatial variable $x$ and stochastic regularity given by a particular weighted chaos space. To compute regular norms of function in the stochastic variable we use the White Noise framework and directional derivatives. This resulting norm require norms of partial derivatives in the $\omega$ variable up to certain order to be bounded. The fact this norm is equivalent to a weighted chaos space norm is proved in Theorem 12.

\section{References}

[1] Ivo Babuška, Raúl Tempone, and Georgios E. Zouraris. Galerkin finite element approximations of stochastic elliptic partial differential equations. SIAM J. Numer. Anal., 42(2):800-825, 2004.

[2] Fred Espen Benth and Jon Gjerde. Convergence rates for finite element approximations of stochastic partial differential equations. Stochastics Stochastics Rep., 63(3-4):313-326, 1998.

[3] Fred Espen Benth and Thomas Gorm Theting. Some regularity results for the stochastic pressure equation of Wick-type. Stochastic Anal. Appl., 20(6):1191-1223, 2002.

[4] D. Berend and T. Tassa. Improved bounds on bell numbers and on moments of sums of random variables. Probability and Mathematical Statistics, 30:185-205, 2010.

[5] Yu. M. Berezanskiǔ. Selfadjoint operators in spaces of functions of infinitely many variables, volume 63 of Translations of Mathematical Monographs. American Mathematical Society, Providence, RI, 1986. Translated from the Russian by H. H. McFaden, Translation edited by Ben Silver.

[6] Vladimir I. Bogachev. Gaussian measures, volume 62 of Mathematical Surveys and Monographs. American Mathematical Society, Providence, RI, 1998.

[7] Ingo Bulla. Dirichlet problem with stochastic coefficients. Stoch. Dyn., $5(4): 555-568,2005$.

[8] Charalambos A. Charalambides. Enumerative combinatorics. CRC Press Series on Discrete Mathematics and its Applications. Chapman \& Hall/CRC, Boca Raton, FL, 2002. 
[9] Julia Charrier. Strong and weak error estimates for the solutions of elliptic partial differential equations with random coefficients. INRIA preprint, http://hal.archives-ouvertes.fr/docs/00/49/67/82/PDF/RR-7300_2_.pdf.

[10] W. G. Cochran, H.-H. Kuo, and A. Sengupta. A new class of white noise generalized functions. Infin. Dimens. Anal. Quantum Probab. Relat. Top., 1(1):43-67, 1998.

[11] Giuseppe Da Prato. An introduction to infinite-dimensional analysis. Universitext. Springer-Verlag, Berlin, 2006. Revised and extended from the 2001 original by Da Prato.

[12] Giuseppe Da Prato and Jerzy Zabczyk. Stochastic equations in infinite dimensions, volume 44 of Encyclopedia of Mathematics and its Applications. Cambridge University Press, Cambridge, 1992.

[13] Philipp Frauenfelder, Christoph Schwab, and Radu Alexandru Todor. Finite elements for elliptic problems with stochastic coefficients. Comput. Methods Appl. Mech. Engrg., 194(2-5):205-228, 2005.

[14] J. Galvis and M. Sarkis. Approximating infinity-dimensional stochastic Darcy's equations without uniform ellipticity. SIAM J. Numer. Anal., 47(5):3624-3651, 2009.

[15] C. J. Gittelson. Stochastic Galerkin discretization of the log-normal isotropic diffusion problem. Math. Models Methods Appl. Sci., 20(2):237263, 2010.

[16] Takeyuki Hida. Brownian motion, volume 11 of Applications of Mathematics. Springer-Verlag, New York, 1980. Translated from the Japanese by the author and T. P. Speed.

[17] Takeyuki Hida, Hui-Hsiung Kuo, Jürgen Potthoff, and Ludwig Streit. White noise, volume 253 of Mathematics and its Applications. Kluwer Academic Publishers Group, Dordrecht, 1993. An infinite-dimensional calculus.

[18] Helge Holden, Bernt Øksendal, Jan Ubøe, and Tusheng Zhang. Stochastic partial differential equations. Probability and its Applications. Birkhäuser Boston Inc., Boston, MA, 1996. A modeling, white noise functional approach.

[19] Hui-Hsiung Kuo. Gaussian measures in Banach spaces. Springer-Verlag, Berlin, 1975. Lecture Notes in Mathematics, Vol. 463.

[20] Hui-Hsiung Kuo. White noise distribution theory. Probability and Stochastics Series. CRC Press, Boca Raton, FL, 1996.

[21] Olga A. Ladyzhenskaya and Nina N. Ural'tseva. Linear and quasilinear elliptic equations. Translated from the Russian by Scripta Technica, Inc. Translation editor: Leon Ehrenpreis. Academic Press, New York, 1968. 
[22] Sergey Lototsky and Boris Rozovskii. Stochastic differential equations: a Wiener chaos approach. In From stochastic calculus to mathematical finance, pages 433-506. Springer, Berlin, 2006.

[23] Sergey V. Lototsky, Boris L. Rozovskii, and Xiaoliang Wan. Elliptic equations of higher stochastic order. M2AN Math. Model. Numer. Anal., 44(5):1135-1153, 2010.

[24] Paul Malliavin. Integration and probability, volume 157 of Graduate Texts in Mathematics. Springer-Verlag, New York, 1995. With the collaboration of Hélène Airault, Leslie Kay and Gérard Letac, Edited and translated from the French by Kay, With a foreword by Mark Pinsky.

[25] A. Mugler and H.-J. Starkloff. On elliptic partial differential equations with random coeffcients. available at http://www.dfgspp1324.de/download/preprints/preprint079.pdf.

[26] Nobuaki Obata. White noise calculus and Fock space, volume 1577 of Lecture Notes in Mathematics. Springer-Verlag, Berlin, 1994.

[27] Luis J. Roman and Marcus Sarkis. Stochastic Galerkin method for elliptic SPDEs: a white noise approach. Discrete Contin. Dyn. Syst. Ser. B, 6(4):941-955, 2006.

[28] Ichiro Shigekawa. Stochastic analysis, volume 224 of Translations of Mathematical Monographs. American Mathematical Society, Providence, RI, 2004. Translated from the 1998 Japanese original by the author, Iwanami Series in Modern Mathematics.

[29] Thomas Gorm Theting. Solving Wick-stochastic boundary value problems using a finite element method. Stochastics Stochastics Rep., 70(3-4):241270,2000 .

[30] Radu Alexandru Todor. Robust eigenvalue computation for smoothing operators. SIAM J. Numer. Anal., 44(2):865-878, 2006.

[31] Ali Süleyman Üstünel. An introduction to analysis on Wiener space, volume 1610 of Lecture Notes in Mathematics. Springer-Verlag, Berlin, 1995.

[32] Xiaoliang Wan. A note on stochastic elliptic model. Comput. Methods Appl. Mech. Engrg., 199(45-48):2987-2995, 2010.

[33] Xiaoliang Wan, Boris Rozovskii, and George Em Karniadakis. A stochastic modeling methodology based on weighted Wiener chaos and Malliavin calculus. Proc. Natl. Acad. Sci. USA, 106(34):14189-14194, 2009.

[34] S. Watanabe. Lectures on stochastic differential equations and Malliavin calculus, volume 73 of Tata Institute of Fundamental Research Lectures on Mathematics and Physics. Published for the Tata Institute of Fundamental Research, Bombay, 1984. Notes by M. Gopalan Nair and B. Rajeev. 